\title{
Recombinant human CD19L-sTRAIL effectively targets B cell precursor acute lymphoblastic leukemia
}

\author{
Fatih M. Uckun, ${ }^{1,2,3}$ Dorothea E. Myers, ${ }^{1}$ Sanjive Qazi, ${ }^{1,4}$ Zahide Ozer, $^{1}$ Rebecca Rose, ${ }^{5}$ Osmond J. D’Cruz, ${ }^{1}$ and Hong Ma \\ 'Children's Center for Cancer and Blood Diseases, Children's Hospital Los Angeles (CHLA), Los Angeles, California, USA. Division of Hematology-Oncology, Department of Pediatrics and \\ ${ }^{3}$ Norris Comprehensive Cancer Center, University of Southern California Keck School of Medicine (USC KSOM), Los Angeles, California, USA. ${ }^{4}$ Bioinformatics Program, Gustavus Adolphus College, \\ St. Peter, Minnesota, USA. ${ }^{5}$ Rose Pathology Services, PLLC, St. Paul, Minnesota, USA.
}

\begin{abstract}
Patients with B cell precursor acute lymphoblastic leukemia (BPL) respond well to chemotherapy at initial diagnosis; however, therapeutic options are limited for individuals with BPL who relapse. Almost all BPL cells express CD19, and we recently cloned the gene encoding a natural ligand of the human CD19 receptor (CD19L). We hypothesized that fusion of CD19L to the soluble extracellular domain of proapoptotic TNF-related apoptosis-inducing ligand (sTRAIL) would markedly enhance the potency of sTRAIL and specifically induce BPL cell apoptosis due to membrane anchoring of sTRAIL and simultaneous activation of the CD19 and TRAIL receptor (TRAIL-R) apoptosis signaling pathways. Here, we demonstrate that recombinant human CD19LSTRAIL was substantially more potent than STRAIL and induced apoptosis in primary leukemia cells taken directly from BPL patients. CD19L-sTRAIL effectively targeted and eliminated in vivo clonogenic BPL xenograft cells, even at femtomolarpicomolar concentrations. In mice, CD19L-sTRAIL exhibited a more favorable pharmacokinetic (PK) profile than sTRAIL and was nontoxic at doses ranging from $32 \mathrm{fmol} / \mathrm{kg}$ to $3.2 \mathrm{pmol} / \mathrm{kg}$. CD19L-sTRAIL showed potent in vivo antileukemic activity in NOD/SCID mouse xenograft models of relapsed and chemotherapy-resistant BPL at nontoxic fmol/kg dose levels. Together, these results suggest that recombinant human CD19L-sTRAIL has clinical potential as a biotherapeutic agent against BPL.
\end{abstract}

\section{Introduction}

B cell precursor acute lymphoblastic leukemia (BPL) is the most common form of cancer in children and adolescents (1-3). Currently, the major challenge in the treatment of BPL is to cure patients who have relapsed despite intensive frontline chemotherapy (4-8). The proapoptotic TNF-related apoptosis-inducing ligand (TRAIL) is a homotrimeric type II transmembrane protein that exhibits potent and selective proapoptotic activity against tumor cells with minimal toxicity to normal cells (9-14). The soluble extracellular domain (ECD) of proapoptotic TRAIL (sTRAIL) triggers apoptosis by binding to its cognate agonistic death receptors TRAIL receptor 1 (TRAIL-R1)/DR4 (Apo2, TNFRSF10A) and TRAIL-R2/DR5 (KILLER, TNFRSF10B). Recombinant human sTRAIL showed a favorable toxicity profile in preclinical studies as well as early clinical trials (15-19). However, (a) a marked heterogeneity in agonistic TRAIL-R expression on cancer cells, coupled with the presence of antagonistic "decoy" receptors TRAIL-R3 (DcR1, TRID, TNFRSF10C), TRAIL-R4 (DcR2, TRUNDD, TNFRSF10D) and osteoprotegerin (OPG, TNFRSF11B) on both cancer cells and normal tissues that compete for sTRAIL binding to cancer cells, (b) the rapid release of sTRAIL from DR4/DR5 due to a rapid off-rate, which is a characteristic feature of ligand-cytokine receptor interactions, and (c) its very short half-life in circulation have been major impediments to its clinical success $(13,16-19)$. It has been proposed that many of these limitations of sTRAIL can be overcome by genetically fusing it to a tumor-specific ligand or antibody $(13,20)$.

Conflict of interest: The authors have declared that no conflict of interest exists Submitted: April 18, 2014; Accepted: December 11, 2014

Reference information: J Clin Invest. 2015;125(3):1006-1018. doi:10.1172/JCI76610.
CD19 is a $95-\mathrm{kDa} \mathrm{B}$-lineage restricted receptor molecule that is expressed on leukemia cells in virtually $100 \%$ of BPL cases, but it is absent on the parenchymal cells of life-maintaining nonhematopoietic organs, circulating blood myeloid and erythroid cells, and T cells as well as bone marrow stem cells (21-23). CD19 has also been found on in vivo clonogenic BPL cells, with leukemia initiating and propagating properties in xenograft models using immunocompromised mice (24-26). The favorable leukemic cell versus normal tissue expression profile of CD19 and its abundant expression on relapse BPL clones make it an attractive molecular target for biotherapy in relapsed acute lymphoblastic leukemia (ALL) (27-31). We recently cloned the gene encoding a natural ligand of the human CD19 receptor (CD19L) from a human thymus cDNA library (31). We hypothesized that a genetic fusion of CD19L to sTRAIL would markedly enhance the potency of sTRAIL and act as a potent inducer of apoptosis for BPL cells due to the membrane anchoring of sTRAIL and the simultaneous activation of the CD19 and TRAIL-R apoptosis signaling pathways. The purpose of the present study was to perform a preclinical evaluation of recombinant human CD19L-sTRAIL fusion protein as a new antileukemic biotherapeutic agent candidate against BPL.

\section{Results}

Heterogeneous expression of surface TRAIL receptors and genetic biomarkers for therapeutic TRAIL sensitivity in primary leukemia cells from BPL patients. Chen et al. recently identified a 71-gene molecular signature that accurately predicted the TRAIL sensitivity of 95 human cancer cell lines (32). Other studies identified CFLAR/ CASPER and TRADD as well as abundant expression of antagonistic TRAIL decoy receptors TRAIL-R3, TRAIL-R4, and TRAIL-R5 
as important predictors of TRAIL resistance (13, 15-17, 20, 33-37). We examined the representation of the TRAIL-sensitivity gene cassette as well as TRAIL death pathway and receptor genes in the transcriptome of primary leukemia cells from newly diagnosed as well as relapsed BPL patients. The BCR-ABL ${ }^{+} / \mathrm{Ph}^{+}, \mathrm{E} 2 \mathrm{~A}-\mathrm{PBX} 1^{+}$, and MLL- $\mathrm{R}^{+}$subsets of BPL carry a high risk of relapse with conventional chemotherapy (38). Notably, 47 of the 68 TRAIL-sensitivity genes (69\%) represented by 82 of the 146 probe sets showed upregulated expression in $\mathrm{BCR}-\mathrm{ABL}^{+} \mathrm{BPL}$ cells, and the $\log _{2}$ robust multiarray analysis (RMA) expression values across the 146 probe sets were $8.02 \pm 0.02$ for leukemia cells from 123 newly diagnosed $\mathrm{BCR}-\mathrm{ABL}^{+} \mathrm{BPL}$ patients versus $7.74 \pm 0.01$ for leukemia cells from 595 newly diagnosed BCR-ABL- BPL patients (pooled SEM from linear contrast model $\left.=0.043, \mathrm{~F}_{1,870}=42.9, P=1 \times 10^{-10}\right)$. The cluster figure in Supplemental Figure 1A (supplemental material available online with this article; doi:10.1172/JCI76610DS1) depicts the expression levels of those TRAIL-sensitivity genes that showed highly significant $(P<0.0001)$ differential expression between BCR-ABL ${ }^{+}$and BCR-ABL- BPL cells (Supplemental Table 1). However, the TRAIL-resistance genes c-FLIP (CFLAR/CASPER) and TRADD were also overexpressed in $\mathrm{BCR}-\mathrm{ABL}^{+}$versus $\mathrm{BCR}-\mathrm{ABL}$ BPL cells (Supplemental Figure 1B). Furthermore, BCR-ABL ${ }^{+}$BPL cells showed upregulated expression of the genes encoding the TRAIL decoy receptors TNF-R3 and TNF-R4 (Supplemental Figure $1 \mathrm{C})$. Thus, BCR-ABL ${ }^{+} \mathrm{BPL}$ cells were characterized by a complex biomarker profile, with representation of both TRAIL-sensitivity and TRAIL-resistance genes in their transcriptome. Primary leukemia cells from the other 2 high-risk subgroups of BPL were also characterized by a complex biomarker profile with representation of both TRAIL-sensitivity and TRAIL-resistance genes in their transcriptome (Supplemental Table 1). No patient subpopulation was identified that showed a uniformly favorable gene expression profile with abundant expression of TRAIL-sensitivity genes, TRAIL-pathway genes, and DR4/DR5 death receptor genes, and low expression levels of TRAIL-resistance-associated genes (Supplemental Table 1). The observed differences between BCR-ABL ${ }^{+}$ versus $\mathrm{E} 2 \mathrm{~A}-\mathrm{PBX} 1^{+}$versus MLL- $\mathrm{R}^{+} \mathrm{BPL}$ cells documented the existence of a marked heterogeneity relative to the genetic biomarkers of TRAIL sensitivity and surface TRAIL-Rs in primary leukemia cells from newly diagnosed BPL patients (Supplemental Table 1). However, each of these 3 high-risk BPL subsets was characterized by a significantly amplified expression of the CD19 receptor gene (BCR-ABL ${ }^{+}$subset: 1.46-fold, $P<0.0001$; E2A-PBX1 ${ }^{+}$subset: 1.79-fold, $P<0.0001$; MLL-R ${ }^{+}$subset: 1.2 -fold, $\left.P<0.0001\right)$. No significant differences were found when the initial biomarker profiles of leukemia cells were compared with those of relapse clones in matched pair diagnosis versus relapse bone marrow specimens from 49 patients with BPL (Supplemental Table 1).

We next used multiparameter flow cytometry to examine the surface-expression levels of CD19 versus the high-affinity agonistic TRAIL-Rs TRAIL-R1/DR4 and TRAIL-R2/DR5 in primary leukemia cells from 21 newly diagnosed patients with BPL (Figure 1). Expression of DR4 was significantly positively correlated with DR5 expression $\left(R^{2}=0.467, P<0.001\right)$. CD10 and CD19 expression levels did not significantly correlate with DR4 or DR5 expression. A significant positive correlation was observed between CD34 and DR5 expression $\left(R^{2}=0.304, P=0.009\right)$, and a positive trend was exhib- ited between CD34 and DR4 expression $\left(R^{2}=0.139, P=0.096\right)$. The mean percentage of $\mathrm{DR} 4^{+}$or $\mathrm{DR}^{+}$leukemia cells was significantly higher in $\mathrm{CD} 34^{+} \mathrm{BPL}$ patients than in CD34- BPL patients. In each patient, only a subpopulation of leukemia cells expressed high enough levels of DR4 (52.2\% $\pm 4.6 \%)$ and/or DR5 (51.2\% $\pm 3.3 \%)$ detectable by above-background fluorescent staining using PE/ FITC-labeled mAb, but CD19 was abundantly expressed on more than $90 \%(93.3 \% \pm 1.3 \%)$ of leukemia cells $(P<0.0001)$ (Figure 1$)$. This finding provided a compelling argument for using CD19L as a CD19 receptor-specific biotargeting element to achieve greater accumulation of STRAIL on the surface membrane of BPL cells and thereby increase the likelihood of an effective crosslinking of the agonistic TRAIL-R1 and TRAIL-R2 receptors.

Selective immunoreactivity and cytotoxicity of recombinant human CD19L-sTRAIL fusion protein. We have successfully cloned and expressed recombinant human CD19L-sTRAIL fusion protein as an antileukemic biotherapeutic agent candidate (Supplemental Figure 2, A-E). CD19L-sTRAIL was purified using anion exchange (AIE) chromatography and size exclusion (SE) chromatography (Supplemental Figure 2F). In this approximately $74-\mathrm{kDa}$ fusion protein, approximately $20 \mathrm{kDa}$ sTRAIL is fused to the C terminus of approximately 54-kDa CD19L protein through a flexible linker (viz, AAAEFAKTTAPSVYPLEPVLESSGSG) (Supplemental Figure 2). The presence of the CD19L and sTRAIL domains was confirmed by Western blot analysis (Supplemental Figure 2, G and H). Fluorescent-labeled CD19L-sTRAIL exhibited selective binding to the surface membrane of CD19+ human leukemia cells, but not to CD19 receptor-negative human 293T cells (Figure 2A). The binding of CD19L-sTRAIL (10 pM) to CD19+ human leukemia cells was mediated via its CD19L domain because it could be blocked by a 100-fold molar excess of an IgG1-Fc fusion protein (1 nM) of recombinant human CD19L used as a CD19 receptorspecific competitor (Figure 2B). When tested side by side at equimolar concentrations, CD19L-sTRAIL showed much stronger binding to CD19+ BPL cells than sTRAIL (Supplemental Figure 3). Even after washing, the cell-bound CD19L-sTRAIL remained stably associated with the surface membrane of CD19+ BPL cells for at least 4 hours, while sTRAIL showed substantially less binding than CD19L-sTRAIL and became rapidly dissociated from the membrane (Supplemental Figure 3). The binding of CD19LsTRAIL to CD19+ human leukemia cells was only minimally affected by the presence of a 50-fold molar excess of sTRAIL (Supplemental Figure 4A). CD19L-sTRAIL caused apoptosis in the CD19 receptor-positive ALL-1 cell line in a concentrationdependent fashion, with approximately $90 \%$ apoptosis at $210 \mathrm{fM}$ and more than $99 \%$ apoptosis at $2.1 \mathrm{pM}$ (Supplemental Figure 5 and Figure 2C). CD19L-sTRAIL-induced (210 fM) apoptosis was mediated by the CD19-specific binding of its CD19L domain to leukemia cells because (a) presence of a 20-fold molar excess of soluble CD19 ${ }^{\mathrm{ECD}}$ protein $(4.2 \mathrm{pM})$ that specifically binds the CD19L domain could significantly block the antileukemic action of CD19L-sTRAIL (33.8\% $\pm 7.3 \%$ apoptosis vs. $87.6 \% \pm 3.5 \%$ apoptosis, $\left.\mathrm{F}_{1,19}=85.4, P<0.0001\right)$, whereas a 20 -fold molar excess of soluble CD19 intracellular domain $\left(\mathrm{CD} 19^{\mathrm{ICD}}\right)$ protein $(4.2 \mathrm{pM})$ that was included as a control protein did not affect its action $(88.9 \% \pm 2.1 \%$ apoptosis vs. $87.6 \% \pm 3.5 \%$ apoptosis) (Figure $2 \mathrm{C}$, $n=4)$ and (b) sTRAIL alone did not cause apoptosis in ALL-1 cells 
A UPN11
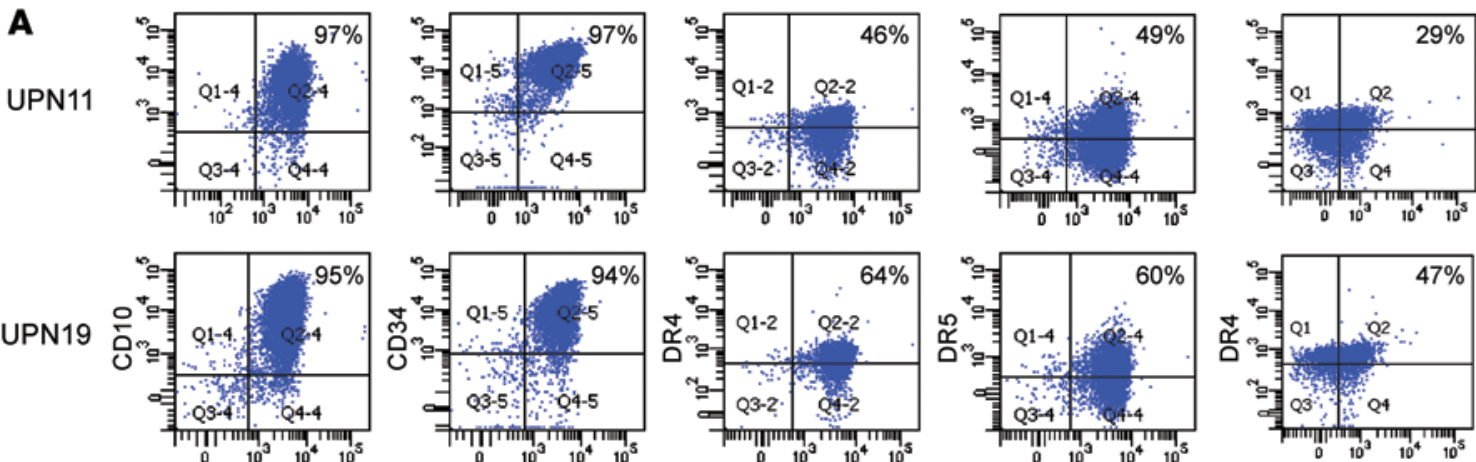

UPN20
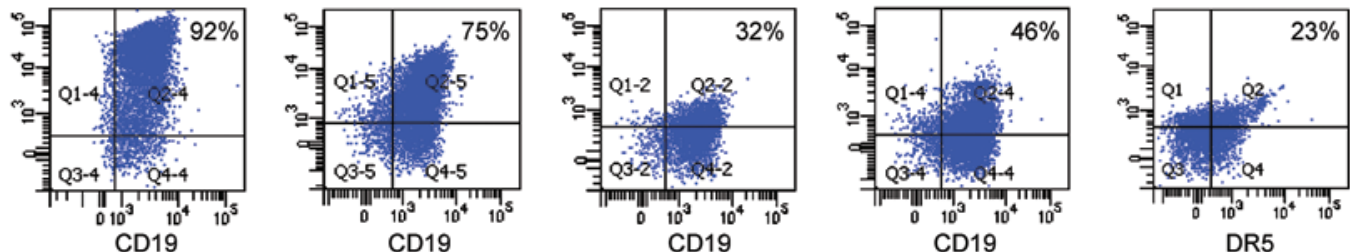

B

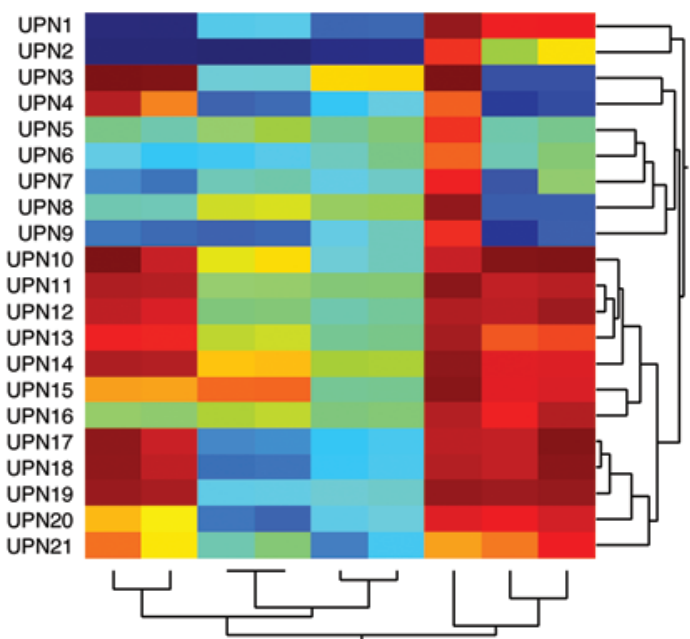

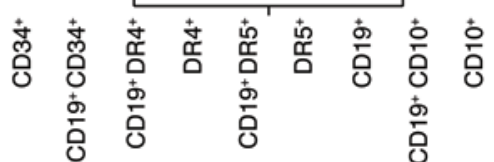

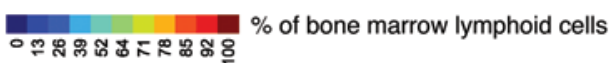

C

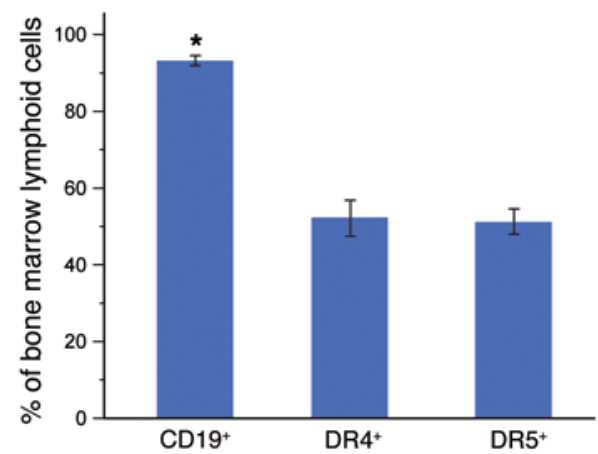

D
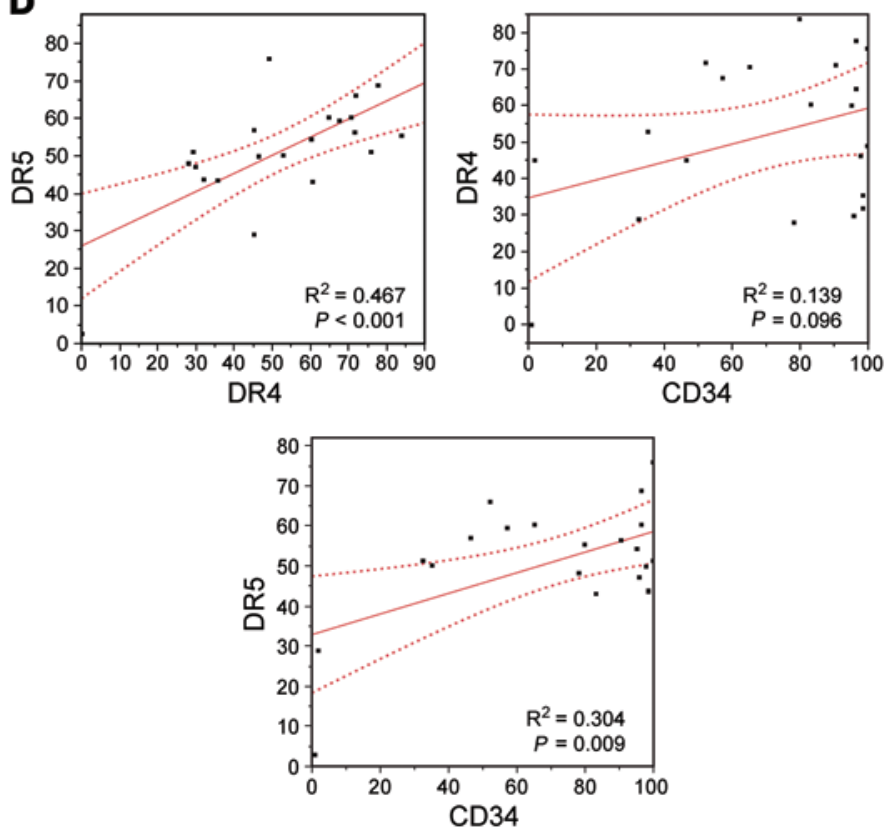

E
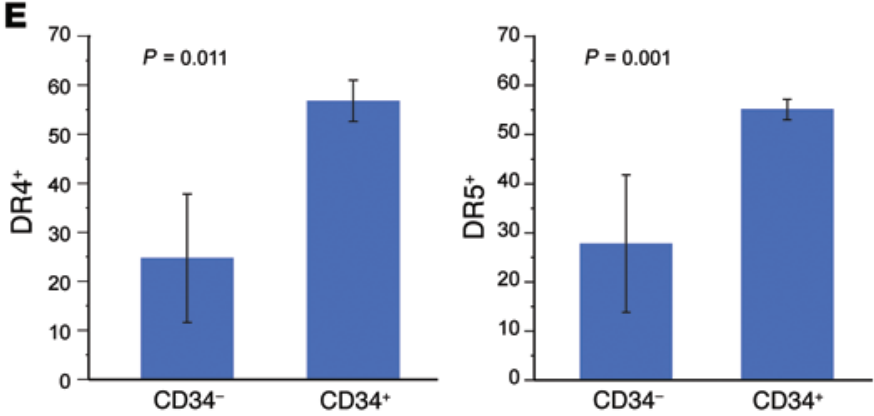
Figure 1. Expression of agonistic TRAIL-Rs TRAIL-R1/DR4 and TRAIL-R2/ DR5 on primary leukemia cells from newly diagnosed pediatric high-risk BPL patients. Leukemia cells from diagnostic bone marrow specimens of 21 high-risk BPL patients were stained by direct immunofluorescence for BPL-associated surface antigens CD10, CD19, and CD34 as well as the death receptors DR4 and DR5. (A) FACS-correlated 2-color fluorescence dot plots of primary leukemia cells from 3 randomly selected representative BPL cases. (B) Cluster figure demonstrating the higher percentage of positivity of leukemia cells for the CD10, CD19, and CD34 antigens versus DR4 or DR5, when examined by direct immunofluorescence and flow cytometry. (C) Depicted are bar graphs comparing the percentage of positivity (mean \pm SEM) for CD19 receptor versus DR4 and DR5 death receptors on primary leukemia cells from all 21 patients shown in B. Significant effect sizes were determined using paired $t$ tests for each patient sample comparing CD19+ versus DR4 ${ }^{+}$or DR5 ${ }^{+}$. ${ }^{*} P<0.0001$ vs. DR4 ${ }^{+}, P<0.0001$ vs. DR5 ${ }^{+}$. (D) Bivariate plots of DR4 versus DR5 exhibited a significant positive correlation in expression of these receptors on primary BPL cells ( $n=21, t$ test on slope parameter, $P=0.0006$ ). Bivariate plots of DR4 and DR5 with CD34 revealed that CD34 was significantly correlated with DR5 expression ( $n=21$,

$P=0.0095$ ) and a trend existed toward a positive correlation with DR4 expression ( $n=21, P=0.096$ ). (E) Depicted are bar graphs comparing the percentage of positivity (mean \pm SEM) for DR4 $(n=21)$ and DR5 $(n=21)$ on primary leukemia cells from CD34+ versus CD34- BPL patients ( $t$ test) using a $30 \%$ positivity cut-off for the immunophenotypic classification.

even at a $100 \mathrm{nM}$ concentration (Supplemental Figure 5). The femtomolar antileukemic potency of CD19L-sTRAIL required the presence of its sTRAIL domain, as soluble CD19L protein alone did not cause apoptosis in ALL-1 cells even at $10 \mathrm{nM}$ (Supplemental Figure 5). Likewise, 2.1 pM CD19L-sTRAIL caused 98.9\% apoptosis in CD19 receptor-positive ALL xenograft cells (BPL xenograft clone no. 11) derived from an E2A-PBX1 fusion transcript-positive relapsed pediatric BPL patient with a $\mathrm{t}(1 ; 19)$ chromosomal translocation, whereas neither $25 \mathrm{nM}$ soluble CD19L nor 250 nM sTRAIL caused apoptosis (Figure 3A).

Activation of the TRAIL death pathway has been shown to trigger the activation of a caspase cascade that is accompanied by a caspase 8 -mediated proteolytic cleavage and activation of the effector caspases 3 and 6, cleavage of poly (ADP-ribose) polymerase (PARP), a substrate for activated caspase 3 , as well as activation of caspase $9(12,13,39)$. Western blot analysis of wholecell lysates from CD19L-sTRAIL-treated ALL-1 cells provided the biochemical documentation of a very rapid TRAIL-R-linked death pathway activation, with emergence of cleavage products of caspase 3, caspase 6, and caspase 9 as well as PARP within 2 hours after initiation of treatment (Figure 2D). There was loss of the cytoskeletal proteins $\alpha$-tubulin and $\alpha / \beta$-actin as well as cleaved caspase proteins in the 4 -hour samples, while the $89-\mathrm{kDa}$ cleaved PARP product remained detectable. The activation of the death pathway in CD19L-sTRAIL-treated ALL-1 cells was blocked by a selective inhibitor of caspase 8 (but not by a selective inhibitor of caspase 9) (Figure 2E), consistent with the prerequisite role of caspase 8 (but not caspase 9) as an initiator caspase in the TRAIL death pathway $(12,39)$. A 50-fold molar excess of sTRAIL (1 nM) was not as effective as CD19L-sTRAIL (20 pM) in activating the TRAIL-R-linked death pathway in CD19L-sTRAIL-treated ALL-1 cells, as documented by detection of the $89-\mathrm{kDa}$ cleaved product of PARP. sTRAIL also did not prevent the PARP cleavage caused by CD19L-sTRAIL (Supplemental Figure 4B). The same conclusion was reached using ALL-1 ${ }^{\text {sT-S }}$ cells, the most sTRAIL-sensitive subclone of the ALL-1 cell line (Supplemental Figure 6A), in which CD19L-sTRAIL not only was a stronger inducer of PARP cleavage, but also caused loss of tubulin within 2 hours, consistent with a rapidly progressive apoptotic process (Supplemental Figure 6B). Furthermore, 210 fM CD19L-sTRAIL caused more apoptosis within 24 hours than sTRAIL did at a $4.8 \times 10^{6}$-fold higher concentration (i.e., $1 \mu \mathrm{M}$ ) (Supplemental Figure 6C). When tested side by side, $210 \mathrm{fM}$ CD19L-sTRAIL was more potent than $1 \mu \mathrm{M}$ sTRAIL, not only against ALL-1 ${ }^{\text {sT-S }}$ cells, but also against the RAJI cell line and the BPL xenograft clone no. 11 as well (Supplemental Figure 7). Even at very high concentrations, standard antileukemic chemotherapy drugs vincristine (VCR) $(11 \mu \mathrm{M})$, dexamethasone (DEX) $(25 \mu \mathrm{M})$, and PEG-asparaginase (10 IU/ml) were not as effective as $210 \mathrm{fM}$ CD19L-sTRAIL (Supplemental Figure 7).

We next examined the effects of CD19L-sTRAIL on normal human B cell precursors and mature B cells using colony-forming assays. The EBV-transformed fetal liver pro-B cell line FL8.2, established from a fetal liver at 8 weeks of gestational age (40), as well as the mature B cell line BCL-1 (41) had lower CD19 expression levels than the BPL cell line ALL-1, as documented by their Alexa Fluor 700-fluorescence intensity differences (Supplemental Figure 8, A-C). ALL-1 cells were DR4-, but strongly DR5+' FL8.2 cells were virtually devoid of DR4 and DR5 receptors, whereas the DR5 expression level of BCL-1 cells was similar to that of ALL-1 cells (Supplemental Figure 8, A-C). FL8.2 cells were resistant to CD19L-sTRAIL (2.1 pM concentration), and BCL-1 cells were less sensitive to CD19L-sTRAIL (2.1 pM concentration) than ALL-1 cells (Supplemental Figure 8D).

In vitro potency of recombinant human CD19L-sTRAIL fusion protein against primary leukemic B cell precursors and leukemic xenograft clones from BPL patients. Primary leukemia cells taken directly from $\mathrm{CD} 19^{+} \mathrm{BPL}$ patients $(n=34)$ were approximately 10 -fold less sensitive to CD19L-sTRAIL than the ALL-1 cell line but were effectively killed at low picomolar concentrations. At a $2.1 \mathrm{pM}$ concentration, CD19L-sTRAIL caused $84.0 \% \pm 4.7 \%$ apoptosis $(n=32)$, whereas ionizing radiation with $2 \mathrm{~Gy} \gamma$-rays caused only $45.0 \% \pm 9.0 \%$ apoptosis $(n=17)(P<0.0001$, Linear contrast $)$ (Figure 3B). More than $90 \%$ apoptosis was achieved at higher concentrations (Figure 3B). Thus, CD19L-sTRAIL was capable of killing radiation-resistant primary leukemia cells at femtomolar to low picomolar concentrations. Likewise, CD19L-sTRAIL caused apoptotic destruction of more than $90 \%$ of very aggressive in vivo clonogenic human ALL xenograft clones isolated from spleens of xenografted NOD/SCID mice that developed overt leukemia after inoculation with primary leukemia cells from BPL patients. Whereas $2.1 \mathrm{pM}$ CD19L-sTRAIL caused $91.4 \% \pm 5.4 \%$ apoptosis $(n=18), 2$ Gy $\gamma$-rays caused only $16.0 \% \pm 4.6 \%$ apoptosis $(n=18)$ $(P<0.0001)$ (Figure 3C).

In vitro potency of recombinant human CD19L-sTRAIL fusion protein against leukemia-initiating ALL xenograft cells derived from $B P L$ patients. Xenograft clones isolated from spleens of NOD/ SCID mice that developed overt leukemia after inoculation with primary leukemia cells from $5 \mathrm{BPL}$ patients were treated for 24 hours at $37^{\circ} \mathrm{C}$ with CD19L-sTRAIL at a concentration of $2.1 \mathrm{pM}$ and then reinjected into NOD/SCID mice. Control NOD/SCID mice were reinjected with xenograft cells that were (a) left untreated for 24 hours at $37^{\circ} \mathrm{C}$, (b) treated with $25 \mathrm{nM}$ soluble recombi- 
A

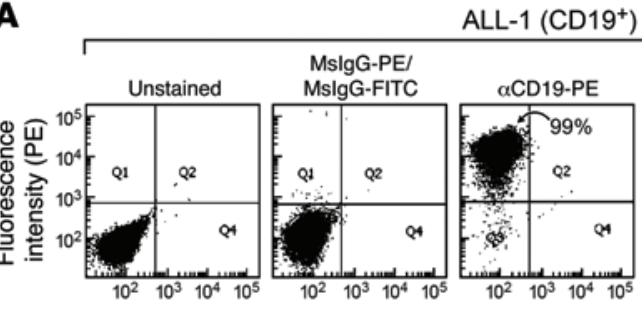

CD19L-STRAIL $\alpha$ CD19-PE/

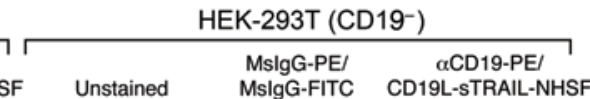
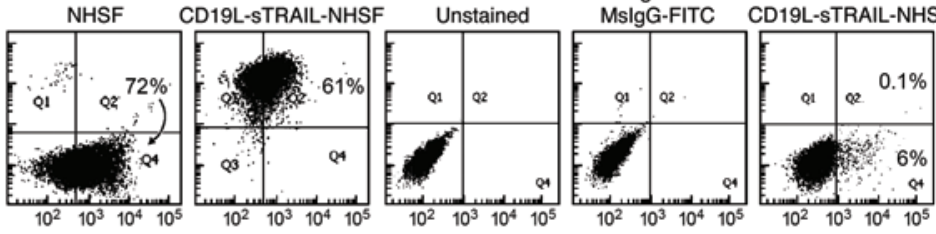

Fluorescence intensity (FITC/NHSF)

B
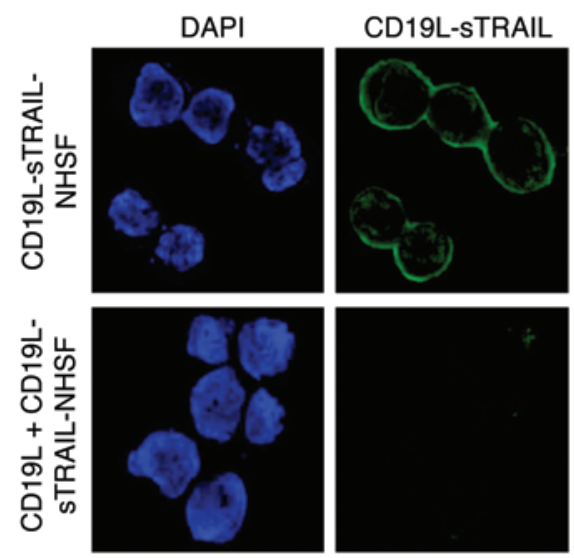
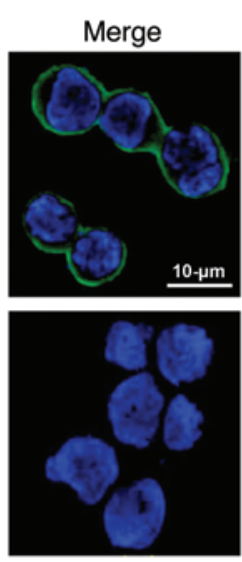

D
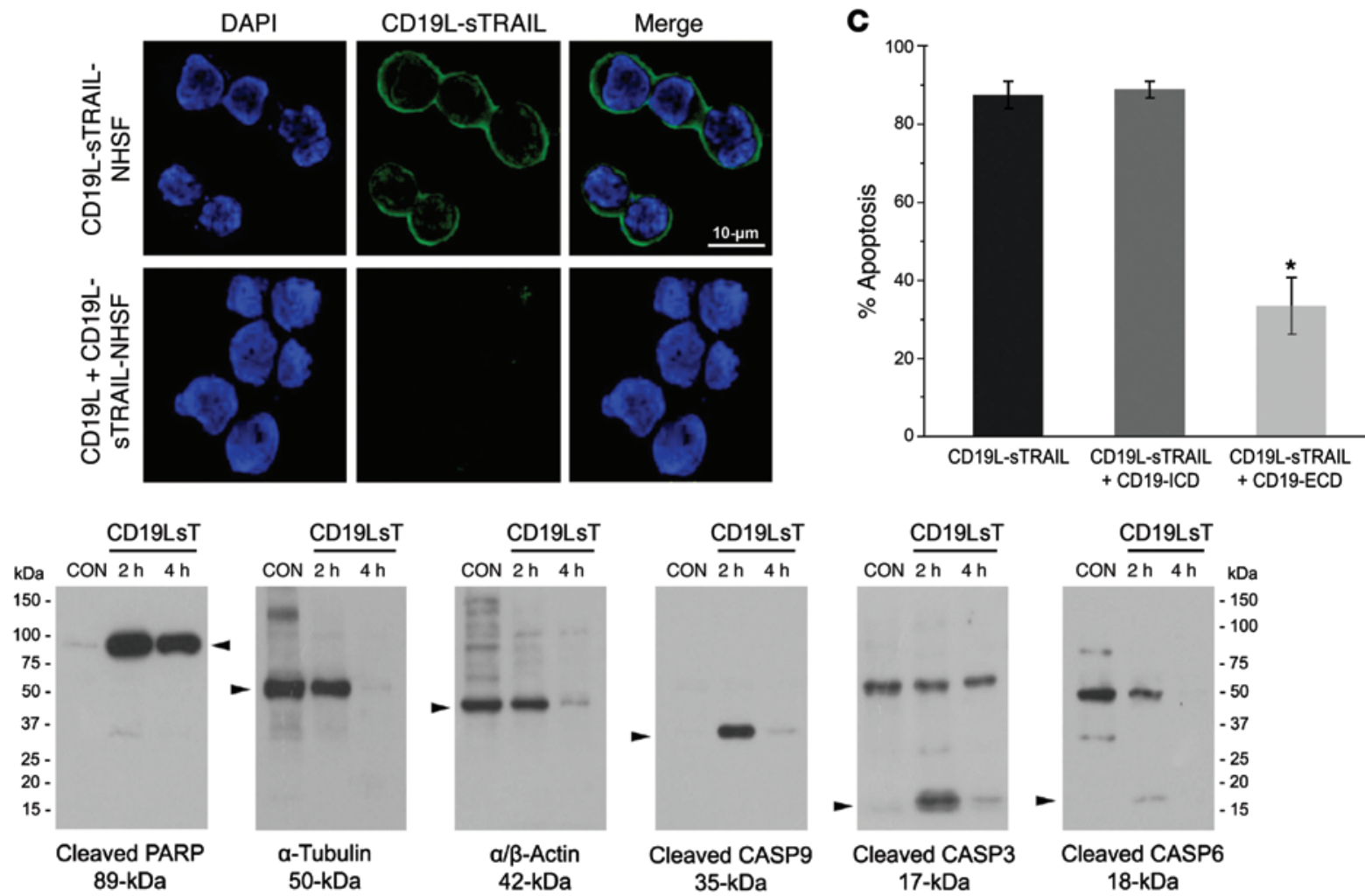

$\mathbf{E}$

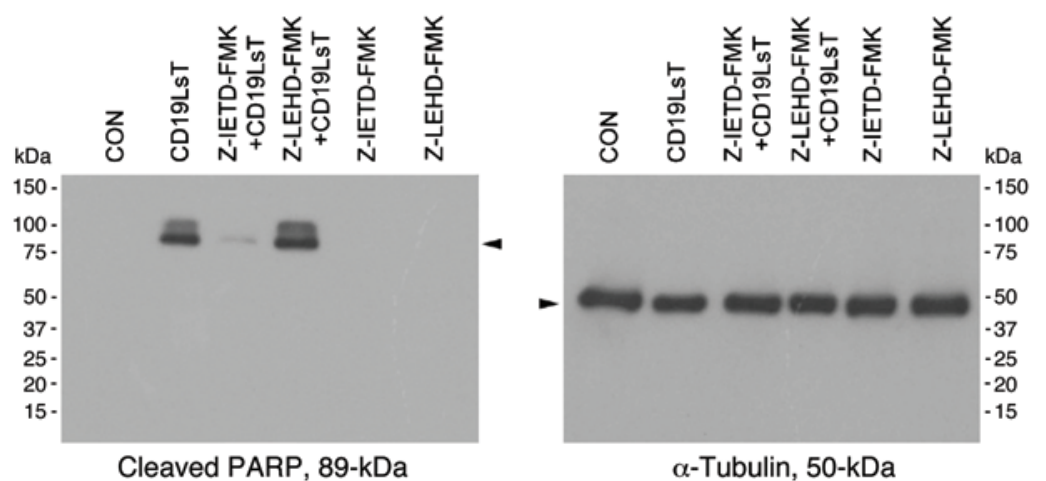

Figure 2. Recombinant CD19L-sTRAIL selectively binds to and kills leukemia cells in a CD19-dependent manner. (A) Two-color fluorescence dot plots showing the immunoreactivity of CD19L-sTRAIL-NHSF (10 pM) with CD19+ ALL-1 cells and its lack of binding to the CD19- HEK-293T negative control cell line. (B) Confocal microscopy images of ALL-1 cells stained with 10 pM NHSF-labeled CD19L-sTRAIL without blocking (upper panels) and with blocking (lower panels) using a 100-fold molar excess of a hlgG1-Fc fusion protein of CD19L (1 nM). (C) ALL-1 cells were treated for 48 hours at $37^{\circ} \mathrm{C}$ with $210 \mathrm{fM}$ CD19LsTRAIL $(n=4), 210 \mathrm{fM}$ CD19L-sTRAIL plus $4.2 \mathrm{pM}(n=4)$ CD19ICD, or $210 \mathrm{fM}$ CD19L-sTRAIL plus $4.2 \mathrm{pM}(n=4)$ CD19ECD. Depicted are bar graphs showing the mean \pm SEM values for the percentage of apoptotic leukemia cells for each treatment group and $P$ values for planned linear contrasts comparing CD19LSTRAIL + CD19 ${ }^{\mathrm{ECD}}$ versus each of the other treatments. ${ }^{*} P<0.001 \mathrm{vs.} \mathrm{other} \mathrm{treatments.} \mathrm{(D)} \mathrm{Depicted} \mathrm{are} \mathrm{Western} \mathrm{blots} \mathrm{documenting} \mathrm{the} \mathrm{TRAIL-R-linked}$ death pathway activation in CD19L-sTRAIL-treated (abbreviated as CD19LsT) (2.1 pM) ALL-1 cells. Controls (CON) included Western blots using anti- $\alpha$ / $\beta$-actin and anti- $\alpha$-tubulin antibodies. (E) Depicted are anticleaved PARP Western blots demonstrating the effects of the selective caspase 8 (CASP8) inhibitor Z-IETD-FMK $(25 \mu \mathrm{M})$ and caspase 9 inhibitor Z-LEHD-FMK $(20 \mu \mathrm{M})$ on TRAIL-R-linked death pathway activation in ALL-1 cells treated with 840 fM CD19L-sTRAIL for 2 hours. The inhibitors were added to the cultures 15 minutes prior to addition of CD19L-sTRAIL. Also shown is the anti- $\alpha$-tubulin Western blot of the same samples. 
A
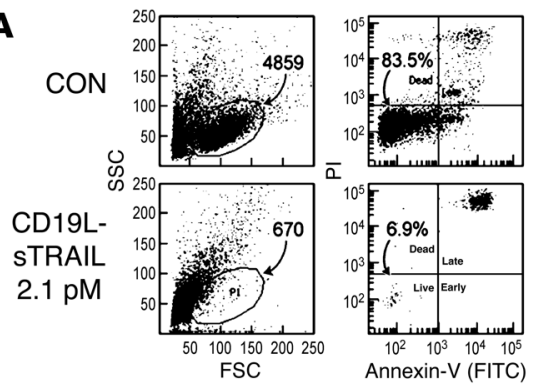

B

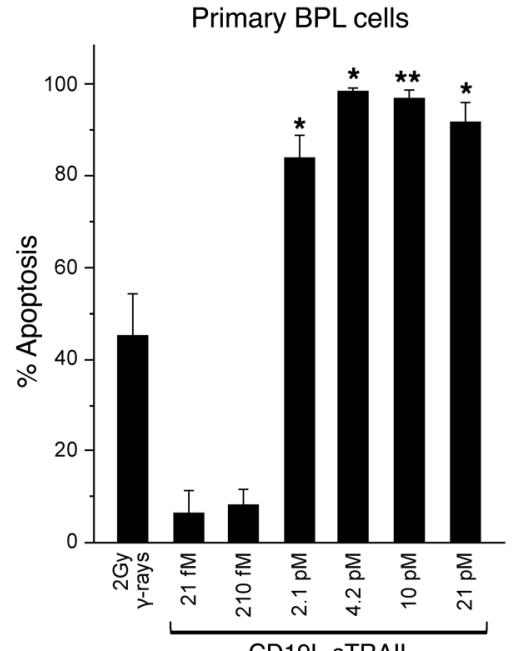

CD19L-STRAIL
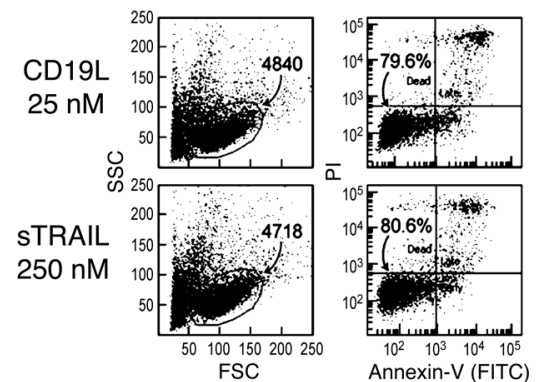

C

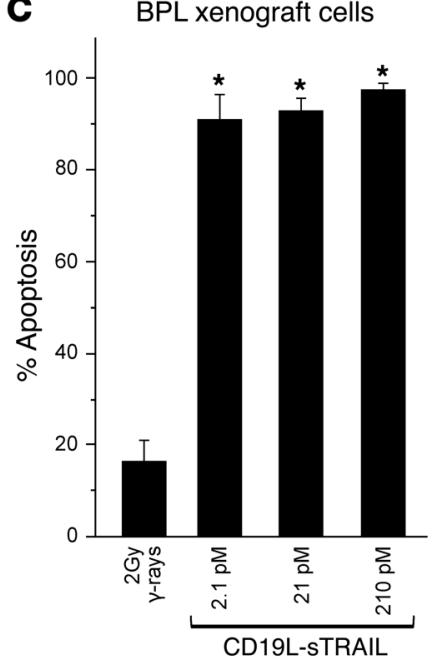

Figure 3. Recombinant human CD19L-sTRAIL causes apoptosis in primary BPL cells and BPL xenograft cells at picomolar concentrations. (A) BPL cells from xenograft clone no. 11 were treated for 48 hours at $37^{\circ} \mathrm{C}$ with CD19L-sTRAIL (2.1 pM), CD19L (25 nM), or sTRAIL (250 nM). Controls included untreated cells. Apoptosis is evidenced by the significantly lower percentages of annexin $\mathrm{V}$-FITC-PI- live cells located in the left lower quadrant of the corresponding 2-color fluorescence dot plots. After CD19L-sTRAIL treatment, cells exhibited marked shrinkage, with altered side scatter (SSC) as well as decreasing numbers of remaining cells in the P1 lymphoid window in the corresponding forward scatter (FSC)/SSC light scatter plot from the 10,000 cells analyzed. (B and C) Primary leukemia cells from 31 newly diagnosed and 3 relapsed patients with BPL and 21 xenograft clones derived from 8 newly diagnosed and 3 relapsed pediatric BPL patients were subjected to CD19L-sTRAIL or 2 Gy $\gamma$-rays. Cells were analyzed for apoptosis as in $\mathbf{A}$. Depicted are bar graphs showing the mean \pm SEM values for the percentage of apoptotic leukemia cells for each treatment group; statistical significance was assessed utilizing planned linear contrasts versus 2 Gy $\gamma$-rays (mixed ANOVA model, with treatment dose as the fixed factor and cell type as a random factor). ${ }^{*} P \leq 0.0001$ vs. $2 \mathrm{~Gy} ;{ }^{* *} P=0.0002$ vs. 2 Gy. Number of samples analyzed in $\mathbf{B}$ :

2 Gy $\gamma$-rays, $n=17 ; 21 \mathrm{fM}, n=2 ; 210 \mathrm{fM}, n=6 ; 2.1 \mathrm{pM}, n=32 ; 4.2 \mathrm{pM}, n=7 ; 10 \mathrm{pM}, n=6 ; 21 \mathrm{pM}, n=7$. Number of samples analyzed in C: 2 Gy $\gamma$-rays, $n=18 ; 2.1 \mathrm{pM}, n=18 ; 21 \mathrm{pM}, n=3 ; 210 \mathrm{pM}, n=3$.

nant human CD19L or $250 \mathrm{nM}$ recombinant human sTRAIL for 24 hours at $37^{\circ} \mathrm{C}$, or (c) irradiated with $200 \mathrm{cGy} \gamma$-rays. All of the 28 control mice challenged with untreated, CD19L-treated or sTRAIL-treated xenograft cells invariably developed fatal leukemia between 25 and 223 days (median $=151$ days: xenograft case no. 1: 110 days; xenograft case no. 2: 151 days; xenograft case no. 3: 223 days; xenograft case no. 10: 125 days; xenograft case no. 11: 25 days). Of 11 mice that were reinjected with irradiated xenograft cells, 5 also developed fatal leukemia within 223 days. Necropsy revealed massive splenomegaly at the time of death in each of these 33 mice (Figure 4). In contrast, only 2 of $24 \mathrm{NOD} /$ SCID mice developed leukemia after reinjection with CD19LsTRAIL-treated xenograft cells (incidence of overt leukemia: 28 of 28 [100\%] mice injected with untreated, CD19L-treated, or
sTRAIL-treated control xenograft cells vs. 2 of 24 [8.3\%] mice injected with CD19L-sTRAILtreated xenograft cells, 2-tailed Fisher's exact test, $P<0.0001 ; 5$ of 11 [45\%] mice injected with irradiated xenograft cells vs. 2 of 24 [8.3\%] mice injected with CD19L-sTRAILtreated xenograft cells, 2-tailed Fisher's exact test, $P=0.0207$ ). The spleens of mice inoculated with untreated xenograft cells measured $2.6 \pm 0.2 \mathrm{~cm}$ and contained an average of 314 $\pm 93 \times 10^{6}$ nucleated cells (Figure 4, A and B). Likewise, the spleens measured $2.4 \pm 0.2 \mathrm{~cm}$ (average nucleated spleen cell count: $180 \pm 54$ $\times 10^{6}$ cells) and $2.2 \pm 0.2 \mathrm{~cm}$ (average nucleated spleen cell count: $200 \pm 45 \times 10^{6}$ cells), respectively, in mice inoculated with CD19L- and sTRAIL-treated xenograft cells (Figure 4, A and B). By comparison, the average values for the spleen size and nucleated spleen cell count were $1.3 \pm 0.1 \mathrm{~cm}$ and $17 \pm 8 \times 10^{6}$ cells, respectively, for the 24 mice challenged with CD19LsTRAIL-treated xenograft cells (Figure 4, A and $B)$. These values were significantly lower than those for mice reinjected with xenograft cells exposed to other treatments or no treatment (linear contrast $P$ values for spleen size: $P<0.0001$ for CD19L-sTRAIL vs. no treatment, $P<0.0001$ for CD19L-sTRAIL vs. other treatments; linear contrast $P$ values for log-transformed nucleated spleen cell counts: $P<0.0001$ for CD19L-sTRAIL vs. no treatment, $P=0.0003$ for CD19L-sTRAIL vs. other treatments). The spleen sizes and nucleated spleen cell counts of mice challenged with CD19L-sTRAIL-treated xenograft cells were very similar to those of control NOD/SCID mice $(n=4)$ that were not inoculated with any leukemia cells (average spleen size: $1.3 \pm 0.1$ $\mathrm{cm}, P=0.91$; average nucleated spleen cell count: $4.5 \pm 0.4 \times 10^{6}$ cells, $\log _{10}$ transformed: $6.65 \pm 0.04, P=0.59)$. Histopathologic examinations were performed in 26 mice challenged with xenograft cells derived from a pediatric E2A-PBX1 $1^{+}$BPL patient who relapsed at 8.8 months after initial diagnosis (viz.: xenograft case no. 11). All 10 of 10 mice receiving untreated cells, 3 of 3 mice receiving CD19L-treated cells, and 3 of 3 mice receiving sTRAIL-treated cells had diffuse leukemic involvement of their bone marrow, meninges, kidney, and/or liver. In contrast, none of the 10 mice receiving CD19L-sTRAILtreated cells had microscopic evidence of leukemic involvement of their bone marrow $(P<0.0001$ vs. control and $P=0.0001$ vs. other treatments, 2-tailed Fisher's exact test), meninges $(P<0.0001$ vs. control and $P=0.0001$ vs. other treatments, 2-tailed Fisher's exact test), kidney $(P<0.0001$ vs. control and $P=0.0014$ vs. other treatments, 2-tailed Fisher's exact test), or liver $(P<0.0001$ vs. control and $P=0.0001$ vs. other treatments, 2-tailed Fisher's exact test) (Figure $4 \mathrm{C}$ ). Thus, at a low picomo- 

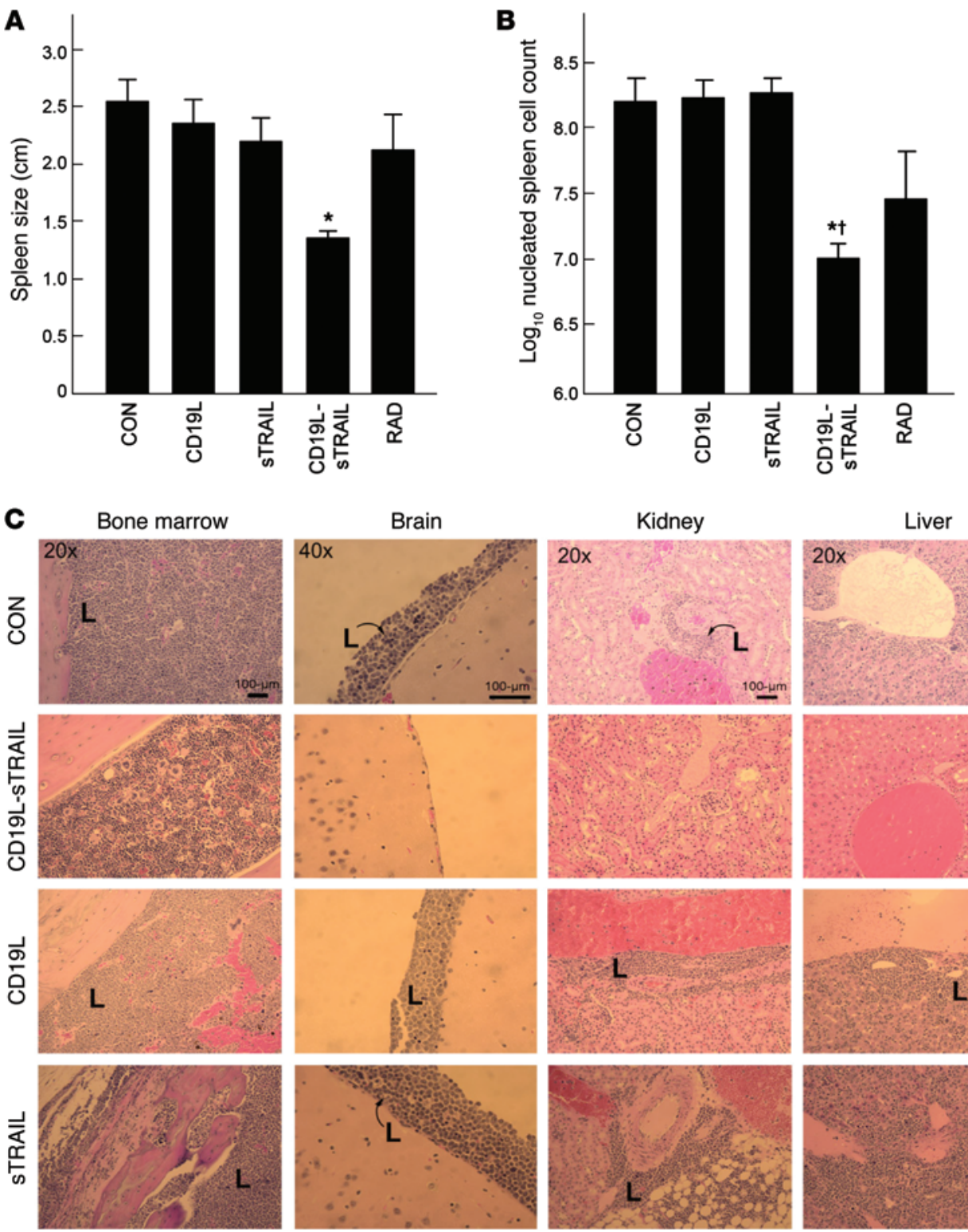

Figure 4. CD19L-sTRAIL abrogates the ability of leukemia-initiating ALL xenograft cells derived from BPL patients to cause leukemia in NOD/SCID mice. ALL xenograft cells $\left(2 \times 10^{6}\right.$ cells/sample) were treated with $2.1 \mathrm{pM}$ CD19L-sTRAIL for 24 hours at $37^{\circ} \mathrm{C}$ or irradiated with 2 Gy $\gamma$-rays. Cells were washed in PBS, resuspended in PBS, and inoculated into NOD/SCID mice i.v. via tail-vein injections in a $100 \mu$ l total volume. Controls $(n=28)$ included untreated xenograft cells as well as xenograft cells treated with CD19L ( $25 \mathrm{nM}$ ) or sTRAIL ( $250 \mathrm{nM})$ for 24 hours at $37^{\circ} \mathrm{C}$ prior to inoculation. Mice were monitored for signs of leukemia, and all mice in a given experiment were sacrificed when any mouse developed morbidity. Histopathologic examinations were performed in 26 mice. (A and B) For each treatment group, the cumulative results for the average spleen size and nucleated spleen cell count, respectively (control: $n=22$ for size, $n=14$ for count); CD19L: $n=3$ for size, $n=2$ for count; sTRAIL: $n=3$ for size, $n=3$ for count; CD19L-sTRAIL: $n=24$ for size, $n=20$ for count;, RAD: $n=11$ for size, $n=10$ for count). SEM and least squares mean estimates for the planned linear contrasts were obtained from mixed ANOVA models. ${ }^{*} P<0.0001$ vs. CON, $P<0.0001$ vs. other treatments in $\mathbf{A} ;{ }^{*} P<0.0001$ vs. CON, ${ }^{\dagger} P=0.0003$ vs. other treatments in $\mathbf{B}$. (C) Mice challenged with untreated, CD19L-treated, or sTRAIL-treated (but not CD19L-sTRAIL-treated) BPL xenograft cells developed disseminated leukemia, with leukemic infiltrates (L) in bone marrow, kidneys, liver, and leptomeninges. Light microscopy images were taken with an EVOS XL Core Light Microscope (AMC Bothel). RAD, radiation.

lar concentration, CD19L-sTRAIL - most likely via apoptotic destruction - was capable of abrogating the ability of the putative leukemic stem cell subpopulation of BPL xenograft cells to initiate leukemia in immunodeficient NOD/SCID mice.
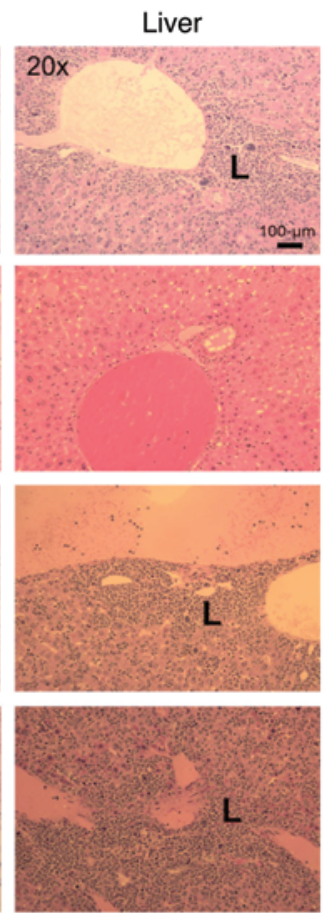

In vivo safety profile, pharmacokinetics, and antileukemic activity of recombinant human CD19L-sTRAIL fusion protein in mice. CD19L-sTRAIL was very well tolerated in C57BL/6 mice without any clinical or laboratory evidence of moderate-severe acute toxicity when administered as a single i.v. bolus dose at 4 different dose levels ranging from $32 \mathrm{fmol} /$ $\mathrm{kg}$ to $3.2 \mathrm{pmol} / \mathrm{kg}$. In particular, blood tests did not reveal any BUN or creatinine elevations suggestive of renal toxicity, alanine transaminase (ALT), aspartate aminotransferase (AST), alkaline phosphatase, lactate dehydrogenase (LDH), or total bilirubin elevations suggestive of hepatic toxicity, amylase/lipase elevations suggestive of pancreas toxicity, or decreases in wbc/rbc/ hemoglobin/platelet count values suggestive of hematologic toxicity. There were no toxic lesions suggestive of any significant parenchymal organ damage (Supplemental Figure 9, A-D, and Supplemental Table 2). We determined the numbers and immunophenotypes of the splenocytes in C57BL/6 mice treated with increasing dose levels of CD19L-sTRAIL in order to determine whether CD19L-sTRAIL causes a depletion of $\mathrm{CD} 19^{+} \mathrm{B}$ cells owing to the $57.5 \%$ identity of the ECD of the mouse and human CD19 proteins. As shown in Supplemental Figure 9E, treatment with CD19L-sTRAIL did not cause a decrease in the abso-

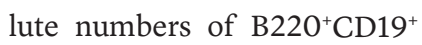
or $\mathrm{CD}^{1} 9^{+} \mathrm{SIgM}^{+} \mathrm{B}$-lineage lymphoid cells.

We next compared the pharmacokinetic (PK) profiles of CD19L-sTRAIL (2.0 pmol/ $\mathrm{kg}$ i.v. bolus) and sTRAIL (6.1 $\mathrm{pmol} / \mathrm{kg}$ ) in C57BL $/ 6$ mice. CD19L-sTRAIL exhibited a favorable PK profile that was superior to the PK profile of sTRAIL. A higher $\mathrm{C}_{\max }$ and a longer plasma half-life characterized the PK profile of CD19L-sTRAIL. Unlike sTRAIL, which was rapidly cleared with 

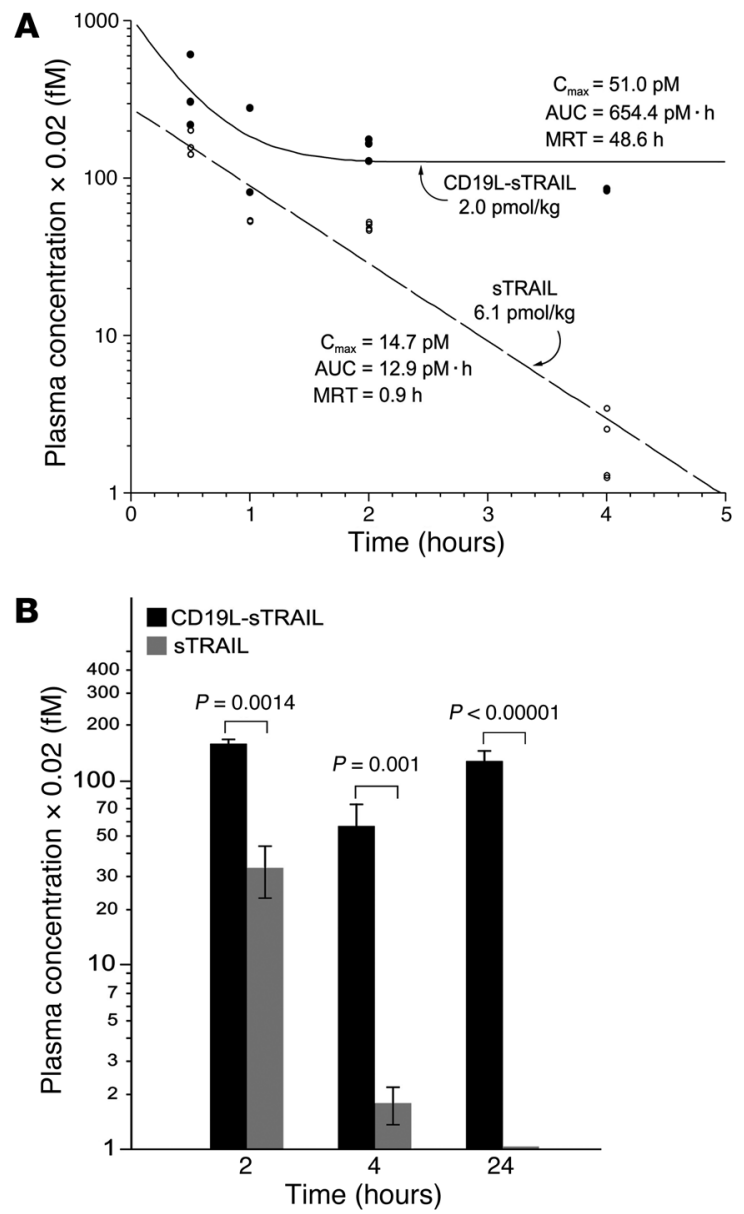

a mean residence time (MRT) of 0.9 hours (plasma half-life $=0.6$ hours), there was little change in plasma CD19L-sTRAIL concentrations between 2 and 24 hours (MRT $=48.6$ hours; plasma halflife $=33.7$ hours) (Figure 5). Despite the lower dose level applied, a significantly higher AUC was achieved with CD19L-sTRAIL $(654.4 \mathrm{pM} \cdot \mathrm{h}$ vs. $12.9 \mathrm{pM} \cdot \mathrm{h})$, consistent with significantly higher plasma concentrations for CD19L-sTRAIL versus sTRAIL at 2 $(P=0.014), 4(P=0.001)$, and 24 hours $(P<0.00001)$. The plasma concentration at 0.5 hours was $17.7 \pm 3.8 \mathrm{pM}$, indicating that the injected $2.0 \mathrm{pmol} / \mathrm{kg}$ bolus dose of CD19L-sTRAIL was distributed into a $113 \mathrm{ml} / \mathrm{kg}$ volume. As this volume is more than twice the average plasma volume in mice, a portion of CD19L-sTRAIL appears to exit the intravascular blood compartment rapidly during the first 2 hours. CD19L-sTRAIL also had a very favorable PK profile in NOD/SCID mice, with an MRT of 43.3 hours (plasma half-life $=33.0$ hours) (calculated using definite integrals for $\mathrm{AUC}_{0-100}$ and $\mathrm{AUMC}_{0-100}$ ) (Figure 6A).

We next examined the in vivo toxicity and antileukemic activity of CD19L-sTRAIL in 2 separate NOD/SCID xenograft models (viz.: xenograft case no. 7 and xenograft case no. 11) of relapsed human BPL. CD19L-sTRAIL was administered i.v. for 2 consecutive days (days 1 and 2) at a nontoxic daily dose level of $17 \mathrm{fmol} / \mathrm{kg} / \mathrm{d}$ (total dose: $34 \mathrm{fmol} / \mathrm{kg}$ ) after administration of an invariably fatal dose of ALL xenograft cells $\left(2 \times 10^{6}\right.$ xenograft cells/mouse $)$ derived from 2 relapsed BPL patients. Single-dose (2 Gy) total body irradiation (TBI) served as a control treatment for comparison. CD19L-sTRAIL
Figure 5. In vivo PKs of CD19L-sTRAIL. C57BL/6 mice were injected with an i.v. bolus dose of $2.0 \mathrm{pmol} / \mathrm{kg}$ CD19L-sTRAIL or $6.1 \mathrm{pmol} / \mathrm{kg}$ sTRAIL in a $100 \mu$ volume. Mice (CD19L-sTRAIL: 2/time point at 1 and 4 hours; 3/time point at $0.5,2$, and 24 hours; sTRAIL: 2/time point at 1, 2, 4 hours; 3/time point at 0.5 and 24 hours) were sacrificed at the indicated time points, and blood samples were collected immediately after $\mathrm{CO}_{2}$ euthanasia. The plasma concentrations of sTRAIL and CD19L-sTRAIL were measured in duplicate by a solid-phase sandwich ELISA using the Human TRAIL/TNFSF10 Quantikine ELISA Kit. (A) The Y-intercept of plasma concentration/time profile determined the $C_{\text {max }}$. The PK parameters $A U C\left(A U C_{0-100 h}\right)$, area under the moment curve ( $\left.A \cup M C_{0-100 h}\right), M R T$, and plasma half-life were calculated as detailed in Supplemental Methods. (B) Depicted are bar graphs showing the mean \pm SEM values and $P$ values for planned linear contrasts (ANOVA model with time and treatment factors, including an interaction term, time $\times$ treatment) for the plasma concentrations of CD19L-sTRAIL versus sTRAIL at 2, 4, and 24 hours after their i.v. administration.

exhibited potent antileukemic activity without any clinical or laboratory evidence of moderate-severe acute toxicity (Supplemental Figure 10). All untreated control mice $(n=10)$ as well as all TBItreated mice $(n=10)$ - but none of the 10 CD19L-sTRAIL-treated mice - developed severe morbidity due to overt leukemia by day 19 (2-tailed Fisher's exact test, $P<0.0001)$. The results were particularly dramatic in xenograft case no. 11 cells derived from a relapsed E2A-PBX1 ${ }^{+}$BPL patient, in which all control mice developed overt disseminated leukemia by day 19 with CNS involvement, massive splenomegaly (average spleen size: $2.5 \pm 0.0 \mathrm{~cm}$; average nucleated spleen cell count of $\left.346.8 \pm 20.0 \times 10^{6}\right)$, and very high numbers of circulating leukemia cells $\left(9.81 \pm 1.2 \times 10^{3} / \mu \mathrm{l}\right)$ (Figure 6, B-D). Notably, the spleen size of CD19L-sTRAIL-treated mice $(n=5)$ was only $1.4 \pm 0.1 \mathrm{~cm}(P=0.0002$ vs. untreated controls; $P=0.003$ vs. TBI), with an average nucleated spleen cell count of $1.7 \pm 0.5 \times 10^{6}$ $(P<0.0001$ vs. untreated controls; $P<0.0001$ vs. TBI). The spleen sizes of mice treated with CD19L-sTRAIL were very similar to those of control NOD/SCID mice $(n=4)$ that were not inoculated with any leukemia cells (average spleen size: $1.3 \pm 0.1 \mathrm{~cm})(P=0.58)$, and their average nucleated spleen cell counts were actually lower than those of control NOD/SCID mice (average nucleated spleen cell count: $4.5 \pm 0.4 \times 10^{6}$ cells; $\log _{10}$ transformed: $6.65 \pm 0.04$ ) $(P=0.01)$. We performed detailed histopathological examination of multiple tissues from each of the 15 mice challenged with these E2A-PBX1 ${ }^{+}$aggressive xenograft BPL cells ( 5 untreated, 5 TBI-treated, and 5 CD19L-sTRAIL-treated mice) (Supplemental Table 3). All of the untreated, but none of the CD19L-sTRAIL-treated mice showed microscopic evidence of disseminated leukemia involving their bone marrow and at least 1 of the other 4 target organ sites examined (viz., lymph node, thymus, brain, spinal cord) (2-tailed Fisher's exact, $P<0.0001)$. In these mice, bone marrow involvement was manifested as replacement of normal tissue elements by diffuse sheets of densely packed leukemia cells. Infiltrated kidneys showed cortical, interstitial, and perivascular accumulations of leukemia cells. Livers showed leukemic infiltrates in the portal spaces and sinusoids. The leptomeninges of the brain contained rafts of leukemia cells. No significant CD19L-sTRAIL-related toxic lesions suggestive of parenchymal organ damage were detected in any of the 18 organs examined.

CD19L-STRAIL fusion protein improves EFS outcome in NOD/ SCID mice with advanced xenografted human BPL. We next used a very aggressive third passage subclone of BPL xenograft clone no. 7 
A
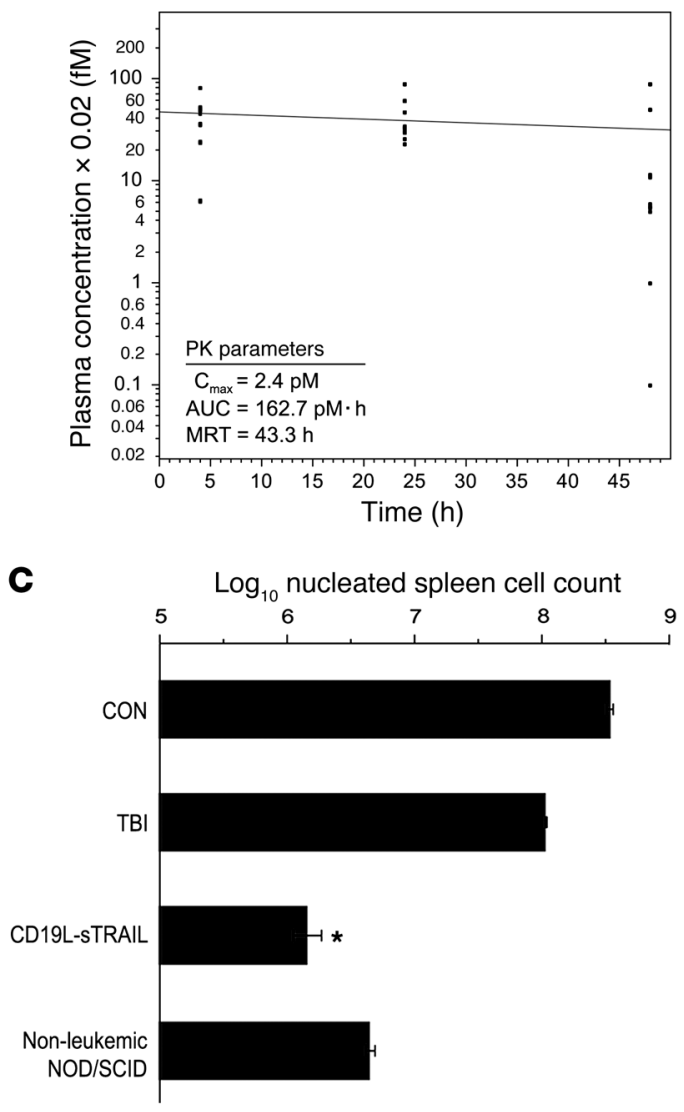

$\mathbf{E}$

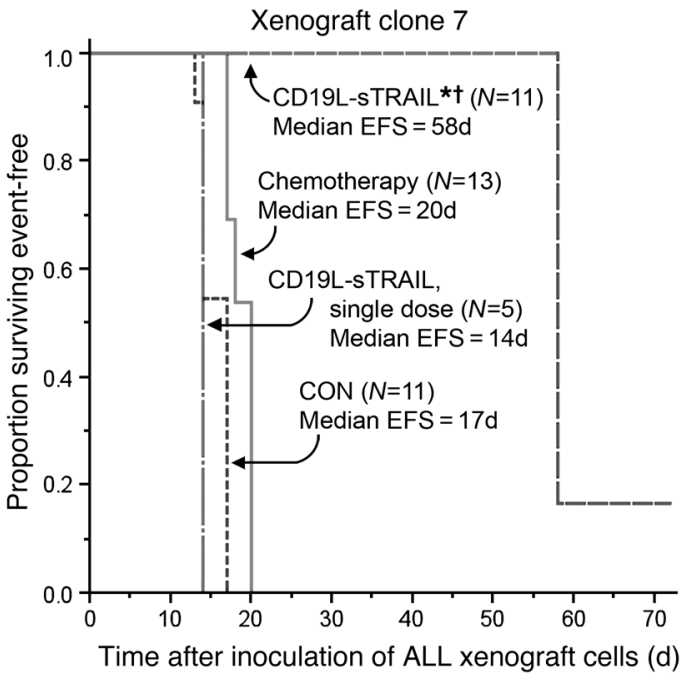

B
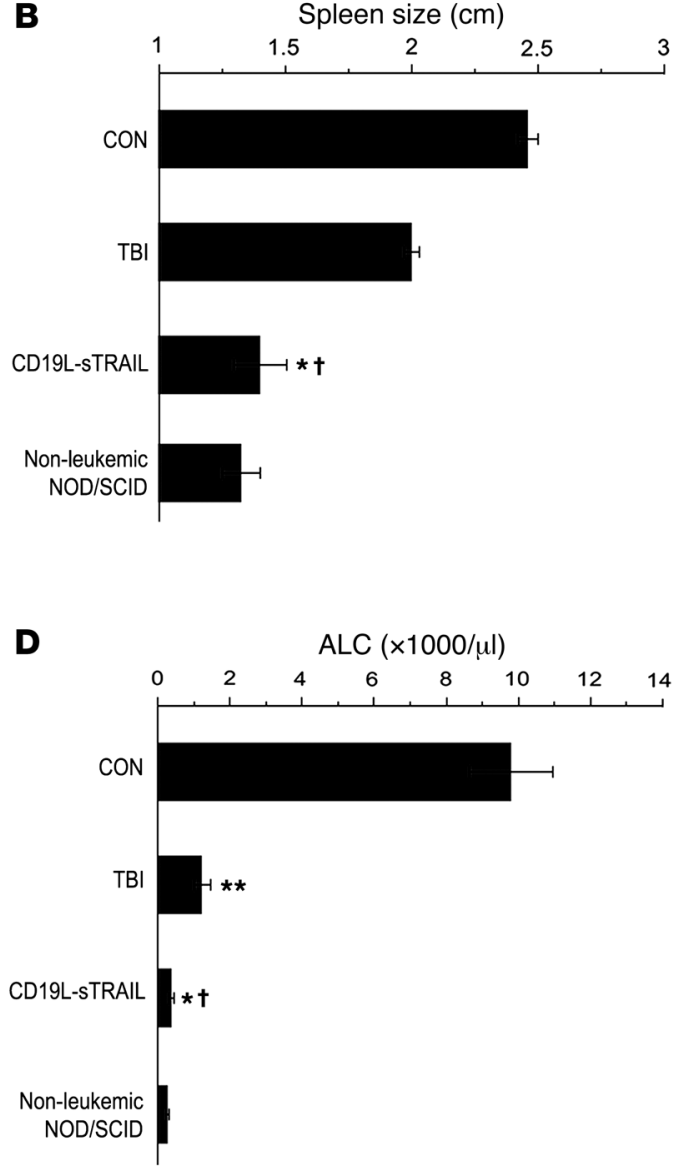

$\mathbf{F}$

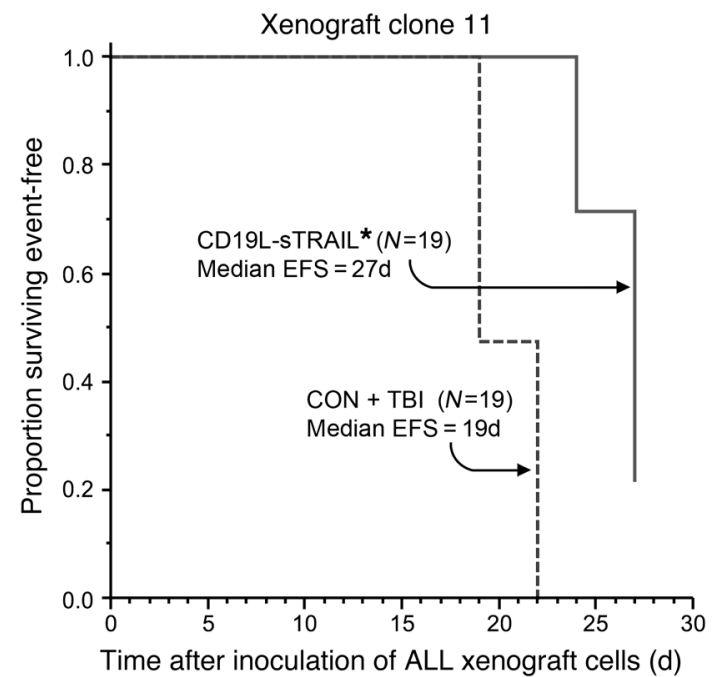

Figure 6. In vivo PKs and antileukemic activity of CD19L-sTRAIL in NOD/SCID mice. (A) NOD/SCID mice were injected i.v. with $2.0 \mathrm{pmol} / \mathrm{kg}$ CD19L-sTRAIL in a $100 \mu \mathrm{l}$ volume. Depicted is the plasma concentration/time curve with the estimated PK parameters ( $n=16$ at 4 hours, $n=16$ at 24 hours, $n=12$ at 48 hours). The PK parameters were calculated as detailed in Supplemental Methods. (B-D) NOD/SCID mice (5/group) were inoculated with $2 \times 10^{6}$ xenograft cells derived from a relapsed BPL patient. CD19L-sTRAIL was administered i.v. on days 1 and $2(17 \mathrm{fmol} / \mathrm{kg} / \mathrm{d})$. One group received 2 Gy TBI on day 2 . The experiment was terminated by euthanasia of all mice in all treatment groups when untreated control mice showed signs of morbidity. Depicted are the mean \pm SEM values ( $n=5$ for control, TBI as well as CD19L-sTRAIL; $n=4$ for nonleukemic control NOD/SCID mice) for the spleen size (B), nucleated spleen cell count (C), and absolute lymphocyte count (ALC) (D). Student's $t$ tests (unequal variances) were performed to compare the control/TBI groups with other groups. ${ }^{*} P=0.0002$ vs. CON, ${ }^{\dagger} P=0.003$ vs. TBI in $\mathbf{B} ;{ }^{*} P<0.0001$ vs. CON or TBI in $\mathbf{C} ;{ }^{*} P=0.0012$ vs. CON, ${ }^{\dagger} P=0.0166$ vs. TBI, ${ }^{*} P=0.0014$ vs. CON in D. (E and F) EFS curves of NOD/SCID mice that were inoculated i.v. with cells from xenograft clone no. 7 and xenograft clone no. $11\left(2 \times 10^{6}\right.$ cells $/$ mouse). For $\mathbf{E}, 11$ control mice challenged with xenograft clone no. 7 were left untreated. For $\mathbf{F}$, among the 19 control mice, 14 challenged with xenograft clone no. 11 were left untreated and 5 received 2 Gy TBI on day 2 . Treatment protocols with CD19L-sTRAIL and standard chemotherapy are detailed in Supplemental Methods. ${ }^{*} P<0.0001$ vs. CON, ${ }^{\dagger} P=0.0002$ vs. chemotherapy in $\mathbf{E} ;{ }^{*} P<0.0001$ vs. CON and TBI in $\mathbf{F}$. $P$ values were calculated utilizing the log-rank test. 

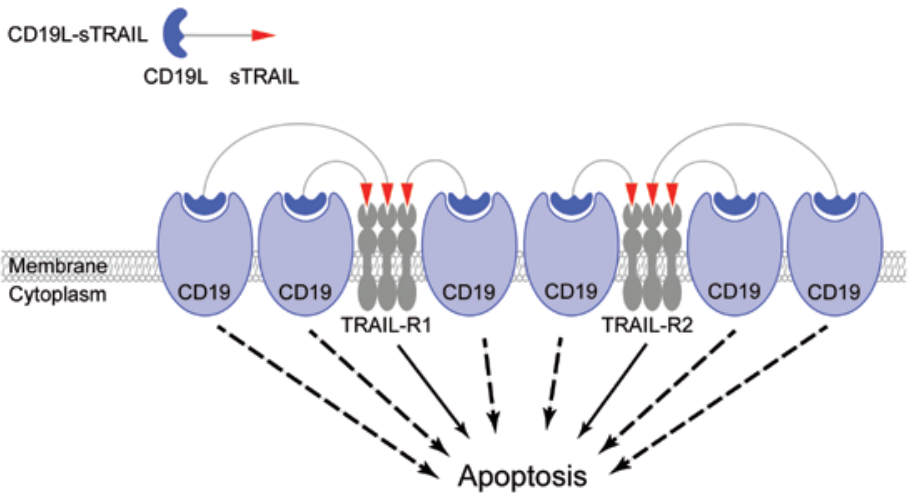

Figure 7. Proposed model for the potent antileukemic activity of CD19L-sTRAIL. sTRAIL is not as active as membrane-bound intact TRAIL in triggering apoptosis after binding to the agonistic TRAIL-Rs TRAIL-R1/DR4 and especially TRAIL-R2/ DR5. Bifunctional sTRAIL fusion proteins engineered with biotargeting ligands have been shown to convert the sTRAIL domain into a much more potent "membrane-anchored" form that mimics TRAIL and is capable of triggering apoptosis via both DR4 and DR5 engagement (see Discussion). Soluble human CD19L protein exhibits exquisite specificity for CD19ECD, perturbs the CD19-associated signaling network, has profound effects on apoptosis-related signaling and gene expression in $\mathrm{CD}^{1} 9^{+}$human leukemia cells, and causes rapid apoptosis in primary leukemia cells from patients with chemotherapy-resistant relapsed BPL at nanomolar concentrations. We propose that the genetic fusion of CD19L to STRAIL depicted at the upper left enhances the potency of sTRAIL due to membrane anchoring and that the engineered 74-kDa bifunctional CD19L-sTRAIL fusion protein acts as a potent inducer of apoptosis for BPL cells due to the simultaneous activation of the CD19 and TRAIL-R apoptosis signaling pathways.

derived from a relapsed BPL patient to examine the in vivo antileukemic potency of CD19L-sTRAIL in a NOD/SCID mouse model of advanced human BPL. In apoptosis assays, these CD19+DR4 $\mathrm{DR}^{+} \mathrm{BPL}$ cells were found to be DEX resistant, PEG-asparaginase resistant, and VCR sensitive (Supplemental Figure 11). Fewer cells survived $210 \mathrm{fM}$ CD19L-sTRAIL than $1 \mathrm{nM}$ sTRAIL, $25 \mu \mathrm{M}$ DEX, $11 \mu \mathrm{M}$ VCR, or $10 \mathrm{IU} / \mathrm{mL}$ PEG-asparaginase (Supplemental Figure 11). Inoculation (i.v.) of NOD/SCID mice with $2 \times 10^{6}$ of these xenograft cells caused rapidly progressive fatal BPL with diffuse bone marrow involvement on day 6, with effacement of normal bone marrow architecture and appearance of circulating leukemia cells in blood. Treatments were initiated on day 10 after inoculation of $2 \times 10^{6}$ xenograft cells unless mice developed signs of leukemia-associated morbidity, including reduced mobility, hunched posture, ruffled fur, or palpable splenomegaly at an earlier time point. All 11 untreated control mice developed fatal leukemia between 13 and 17 days, with a median event-free survival (EFS) time of only 17 days (Figure 6E). Treatment with combination chemotherapy regimens VCR $(0.05 \mathrm{mg} / \mathrm{kg})$ plus doxorubicin $(2 \mathrm{mg} / \mathrm{kg})$ plus PEG-asparaginase (850 IU $/ \mathrm{kg})(n=6)$ or VCR $(0.05 \mathrm{mg} / \mathrm{kg})$ plus DEX (2 mg/kg) plus PEG-asparaginase (850 $\mathrm{IU} / \mathrm{kg})(n=7)$ resulted in a statistically significant but only 3-day prolongation of the median EFS time to 20 days ( $n=13, P$ vs. control $=0.0001)$. Likewise, treatment of mice with a single dose of $17 \mathrm{fmol} / \mathrm{kg}$ CD19L-sTRAIL did not improve the EFS outcome $(n=5$, median $\mathrm{EFS}=14$ days, $P$ vs. control $=0.1)$. In contrast, $2-$ to 3 -day treatment with CD19L-sTRAIL as a single agent $(24 \mathrm{fmol} / \mathrm{kg}$ over 2 days with commencement on day $6: n=5 ; 51 \mathrm{fmol} / \mathrm{kg}$ over 3 days with commencement on day 10: $n=6$ ) significantly improved
EFS outcome and prolonged the median EFS time to 58 days $(P$ vs. control $<0.0001 ; P$ vs. chemotherapy $=0.0002 ;$ Figure 6E). CD19L-sTRAIL (34 fmol/ $\mathrm{kg}$ ) administered as a single dose on day $10(n=14)$ or in 2 doses on days 1 and $2(n=5)$ also improved EFS outcome of NOD/SCID mice challenged with xenograft clone no. 11 (median EFS = 19 days for control mice vs. 27 days for CD19L-sTRAIL-treated test mice, $P<0.0001$ ) (Figure 6F). These results provided the preclinical proof of concept that CD19L-sTRAIL may be effective in the treatment of chemotherapy-resistant relapsed BPL.

\section{Discussion}

Soluble recombinant human CD19L protein exhibits exquisite specificity for the ECD of CD19, perturbs the CD19-associated signaling network, has profound effects on apoptosis-related signaling and gene expression in $\mathrm{CD}_{1}{ }^{+}$human leukemia cells, and causes rapid apoptosis in primary leukemia cells from patients with chemotherapy-resistant relapsed BPL at nanomolar concentrations (31). We hypothesized that a genetic fusion of recombinant human CD19L to sTRAIL would enhance the potency of sTRAIL due to membrane anchoring and that the engineered $74-\mathrm{kDa}$ bifunctional recombinant human CD19L-sTRAIL fusion protein would be a potent inducer of apoptosis for BPL cells due to the simultaneous activation of the CD19 and TRAIL-R apoptosis signaling pathways (Figure 7). As the binding of CD19L-sTRAIL to CD19+ human leukemia cells would be mediated via its CD19L domain, the use of the fusion protein was also anticipated to result in a much higher and more stable accumulation of the sTRAIL domains on the surface and thereby provide an opportunity for them to more effectively engage the TRAIL-Rs. Furthermore, CD19L-sTRAIL would exhibit a more favorable PK profile than 20-kDa sTRAIL because of its larger size and more restricted biodistribution. In agreement with our hypotheses, CD19L-sTRAIL selectively caused apoptotic death in CD19 receptor-positive leukemia cells at femtomolar-picomolar concentrations. It was more than 5 logs more potent than recombinant human sTRAIL and more than 3 logs more potent than recombinant human CD19L proteins alone, which were also tested in side-by-side comparison. CD19L-sTRAIL was very well tolerated in C57BL/6 mice at dose levels ranging from $32 \mathrm{fmol} / \mathrm{kg}$ to $3.2 \mathrm{pmol} / \mathrm{kg}$. At a nontoxic dose level of $17 \mathrm{fmol} / \mathrm{kg}$ administered i.v. only on 2 to 3 consecutive days, CD19L-sTRAIL showed promising in vivo antileukemic activity in NOD/SCID mouse xenograft models of relapsed BPL. CD19LsTRAIL exhibited (a) potent in vitro antileukemic activity at $3 \mathrm{log}$ lower concentrations and (b) potent in vivo antileukemic activity at $3 \log$ lower dose levels than scFvCD19: sTRAIL, another CD19specific sTRAIL fusion protein (Mr: $47 \mathrm{kDa}$ ) in which the scFv of an anti-CD19 mAb was genetically linked to sTRAIL (42). In contrast to CD19L-sTRAIL and scFvCD19:sTRAIL, even nanomolar concentrations of recombinant sTRAIL induced apoptosis in less than $25 \%$ of BPL cases (37). In the present study, high nanomolar concentrations of sTRAIL did not cause apoptosis of BPL cells or affect the ability of leukemia-initiating cells in BPL xenograft specimens to engraft and cause fatal leukemia in NOD/SCID mice.

The PK profile of sTRAIL is characterized by a very short plasma half-life (15). An IgG-Fc domain fusion protein of sTRAIL was 
reported to have a modestly prolonged plasma half-life of $100 \mathrm{~min}$ utes (43). Despite its larger size, which should limit its renal clearance, that Fc fusion protein was likely cleared by the Fc receptorpositive elements of the reticuloendothelial system. As expected, the sTRAIL-Fc fusion protein exhibited 5 -fold improved antitumor activity over sTRAIL when tested at a $152 \mathrm{nmol} / \mathrm{kg}$ dose level (43). As CD19L-sTRAIL has a molecular weight of $74 \mathrm{kDa}$ and does not have an Fc-like domain, which would cause it to be cleared by the reticuloendothelial system, we expected its in vivo elimination to be very slow. In accordance with this expectation, the estimated plasma half-life of i.v.-administered CD19L-sTRAIL in C57BL/6 mice was 33.7 hours, whereas the plasma half-life of sTRAIL in a side-by-side comparison was 0.6 hours. The longer plasma halflife of CD19L-sTRAIL was associated with a significantly superior systemic exposure level (viz.: AUC) when compared with sTRAIL. CD19L-sTRAIL also had a favorable PK profile in NOD/SCID mice, with an estimated plasma half-life of 30 hours. Its favorable PK profile and its CD19-restricted binding to cells were expected to contribute to a favorable in vivo bioavailability of CD19L-sTRAIL for CD19 ${ }^{+}$target leukemia cells. CD19L-sTRAIL showed potent in vivo antileukemic activity in NOD/SCID mouse xenograft models of relapsed BPL at nontoxic fmol $/ \mathrm{kg}$ dose levels. We attribute the observed in vivo antileukemic potency of CD19L-sTRAIL at the $\mathrm{fmol} / \mathrm{kg}$ dose levels to both its favorable in vivo bioavailability and its picomolar activity as a potent and CD19 receptor-selective apoptosis inducer. The ability of CD19L-sTRAIL as a single agent to cause destruction of primary leukemia cells and xenograft cells derived from relapsed BPL patients both in vitro and in vivo indicates that its further development may provide the basis for new and more effective treatment strategies against therapy-refractory or relapsed BPL. CD19L-sTRAIL could represent an important addition to the emerging new personalized treatment options for BPL, including cell-based targeted immunotherapy using autologous T cells expressing CD19-specific chimeric antigen receptors (29). Recombinant human CD19L-sTRAIL fusion protein was overall very well tolerated in mice without any clinical or laboratory evidence of moderate-severe acute toxicity at therapeutically effective dose levels, and post-mortem histopathological examinations revealed no toxic lesions suggestive of significant parenchymal organ damage in any of the organs examined. Nevertheless, the risk of immunosuppression due to a CD19L-sTRAIL-mediated B cell depletion, with an increased susceptibility to infections, is a real possibility, as with any biotherapeutic agent targeting antigens expressed on B cells.

The efficacy of the fusion protein does not seem to be limited by factors that have been shown to limit the action of sTRAIL. Although our biomarker analyses did not reveal a uniformly favorable gene expression profile with abundant expression of TRAIL-sensitivity genes and low expression levels of TRAILresistance-associated genes for any BPL patient population, primary leukemia cells from each of $34 \mathrm{BPL}$ patients as well as 21 BPL xenograft clones from 11 patients were uniformly sensitive to low concentrations of CD19L-sTRAIL. Sixty molecules of CD19LsTRAIL/target leukemia cells (210 fM concentration) caused $90 \%$ apoptosis, and 600 molecules of CD19L-sTRAIL/target leukemia cells (2.1 pM concentration) caused greater than 99\% apoptosis. No single case was identified that showed de novo CD19L-sTRAIL resistance. Furthermore, blocking experiments with CD19L, CD19-ECD, or sTRAIL have demonstrated that the binding of CD19L-sTRAIL to leukemia cells is mediated predominantly via its CD19L domain. Therefore, the presence of decoy TRAIL-Rs on normal tissues should not emerge as an obstacle for CD19LsTRAIL. However, these promising results should be interpreted with due caution, as CD19-negative leukemic subclones may be encountered in clinical settings and would almost certainly escape the lethal action of CD19L-sTRAIL. There may also be as-yetunknown host pharmacogenomic biomarkers that may adversely influence the plasma and tissue stability of CD19L-sTRAIL in patients, thereby affecting its clinical efficacy. Ultimately, the biomarkers of resistance for CD19L-sTRAIL will require correlated laboratory investigations in clinical trial settings.

\section{Methods}

Recombinant human CD19L-sTRAIL fusion protein. We used a stepwise molecular cloning strategy using the commercially available pFUSEhIgG1-Fc2 plasmid (Invivogen) as the backbone vector to construct an expression cassette for CD19L-sTRAIL (see Supplemental Methods for a detailed description of the methodology). Recombinant human CD19L, CD19 ${ }^{\mathrm{ECD}}$, and CD19 ${ }^{\mathrm{ICD}}$ proteins were prepared as previously reported (31) and used as controls. We also used a hIgG1-Fc fusion protein of CD19L in some blocking experiments to confirm that the binding of the fluorescent-labeled CD19L-sTRAIL fusion protein to leukemia cells is mediated via its CD19L domain (see Supplemental Methods).

Gene expression profiling, immunophenotyping, immunofluorescence microscopy, Western blot analyses, and apoptosis assays. Gene expression profiling, immunophenotyping using $\mathrm{mAb}$, confocal imaging, immunoblotting using the ECL detection system (Amersham Pharmacia Biotech), and apoptosis assays were performed, as described in detail in previous publications (40, 41, 44-46) and Supplemental Methods. For bioinformatics analyses of gene expression profiles, we used archived data sets on primary leukemia cells from matchedpair relapse versus diagnosis leukemia specimens from relapsed BPL patients as well as newly diagnosed BPL patients, including those with Philadelphia chromosome (Ph)/BCR-ABL fusion transcript-positive, $\mathrm{t}(1 ; 19)$ /E2A-PBX1 fusion transcript-positive, or MLL-rearrangement (R)-positive disease, as detailed in Supplemental Methods.

Leukemia cells. Primary leukemia cells isolated from bone marrow $(n=28)$ or peripheral blood $(n=6)$ specimens of 34 patients with BPL (new diagnosis $=31$; relapse $=3$ ) and 21 clones derived from spleen specimens of 21 xenografted NOD/SCID mice inoculated with leukemia cells from 11 BPL xenograft cases were used in the described experiments. Of these 21 clones, 3 were derived from xenograft case no. 7, 5 were derived from xenograft case no. 8, 2 were from xenograft case no. 10, and 4 were from xenograft case no. 11. The remaining 7 clones were from xenograft case nos. 1, 2, 3, 4, 5, 6, and 9. The 11 xenograft cases were established using primary cells from 8 newly diagnosed and 3 relapsed pediatric BPL patients. We also used the ALL-1 $\left(\mathrm{Ph}^{+}\right.$adult BPL), RAJI (Burkitt's leukemia/B-ALL) (44), FL8.2 normal human pro-B cell (40), and BCL-1 normal mature B cell lines (41).

Preclinical studies of CD19L-sTRAIL in rodents. The toxicity and PKs of CD19L-sTRAIL were studied in mice using previously published methods (46). The antileukemic activity of CD19L-sTRAIL was studied in a NOD/SCID mouse model of human BPL. See Supplemental Methods for a description of these methodologies. 
Statistics. Standard methods were used for statistical analysis of data, as detailed in Supplemental Methods. In particular, a linear model was used for the analysis of the apoptosis data. We constructed 2 mixed models of ANOVA utilizing RMA-normalized gene expression levels to identify significant differences among leukemia patients. We then used a supervised approach with planned linear contrasts to assess significant effect sizes in probe set-specific and gene set-specific comparisons. Pair-wise Pearson product-moment correlations were performed between DR4, DR5, CD19, and CD34 expression levels in primary leukemia cells. Least squares regression analysis was utilized to determine the fraction of variation explained by the Pearson correlation, and significance of the association was tested against 0 correlation using the $\mathrm{F}$ test for regression. $P$ values of less than 0.05 were deemed significant. For the analysis of PK data, we performed specific comparisons of the plasma concentrations of sTRAIL versus CD19L-sTRAIL at each time point using a planned linear contrast model for $\log _{10}$-transformed values, and in this model, values below 0 were floored at a value of 1 . The statistical model included 2 fixed effects (time and formulation) and an interaction term (time $\times$ formulation). The least squares method was used to fit the parameters for the general linear model, and these parameters were utilized to generate prediction equations and best fit lines visualized by plotting leverage graphs using standard coding procedures. We examined the distribution of the residuals of the model for equal dispersion around the line of best fit. Effect sizes were calculated from differences between the least squares mean estimates of the respective plasma concentrations for sTRAIL and CD19L-sTRAIL (coded 1 and -1 in the specified contrasts). The effect sizes were used in the design of planned linear contrasts in order to determine the significance of the observed differences in plasma concentrations at the indicated time points. The differences were deemed significant for 2-tailed $P$ values of less than 0.05 in the JMP software (SAS). For the evaluation of the in vivo antileukemic activity of CD19L-sTRAIL, the probability of survival was determined and the event-free interval curves were generated using the Kaplan-Meier product limit method. To compare differences in median survival estimates between all groups and pairwise comparison of pooled controls versus CD19L-sTRAIL-treated mice, log-rank tests were performed. For the analysis of the in vitro potency of CD19LsTRAIL against leukemic stem cells in xenograft specimens, 2-tailed $t$ tests with correction for unequal variance (Microsoft Excel) were performed comparing various parameters of leukemic burden, including the mean spleen size and cellularity for the various treatments.

Study approval. The animal research in mice was conducted according to approved IACUC (CHLA) protocols 280-09/280-12 and 293-10/293-13. All animal care procedures conformed to the Guide for the Care and Use of Laboratory Animals (National Research Council, National Academy Press, Washington DC, USA. Revised 1996). Leukemia cells isolated from deidentified patient specimens were used in the described experiments. The secondary use of leukemia cells for subsequent laboratory studies did not meet the definition of human subject research per 45 CFR 46.102 ( $d$ and f), since it did not include identifiable private information, and the corresponding research protocol CCI-10-00141 was approved by the CHLA IRB (CCI) (human subject assurance number FWA0001914).

\section{Acknowledgments}

The project described was supported by a 2011 V-Foundation Translational Research Award (to F.M. Uckun) and in part by Department of Health and Human Services grants R21CA-164098, U01-CA-151837, and R01-CA-154471 (to F.M. Uckun) from the National Cancer Institute. The content is solely the responsibility of the authors and does not necessarily represent the official views of the National Cancer Institute or the NIH. We thank Martha Arellano, Erin Feinstone, Cherish Flowers, Anoush Shahidzadeh, Ingrid Cely, and Erika Olson from the Uckun Lab at the Children's Center for Cancer and Blood Diseases/CHLA for technical assistance. We further thank Parvin Izadi of the CHLA Bone Marrow Laboratory and Tsen-Yin Lin of the CHLA FACS Core as well as Ernesto Barron and Anthony Rodriguez of the USC Norris Comprehensive Cancer Center Cell and Tissue Imaging Core for their assistance. We also thank Amanda Termuhlen and Paul Gaynon for providing primary BPL specimens.

Address correspondence to: Fatih M. Uckun, Children's Hospital Los Angeles, MS\#160, Los Angeles, California 90027-0367, USA. Phone: 323.361.4328; E-mail: fmuckun@chla.usc.edu or uckun@usc.edu.
1. Pui CH, Mullighan CG, Evans WE, Relling MV. Pediatric acute lymphoblastic leukemia: where are we going and how do we get there? Blood. 2012;120(6):1165-1174.

2. Trigg ME, et al. Ten-year survival of children with acute lymphoblastic leukemia: a report from the Children's Oncology Group. Leuk Lymphoma . 2008;49(6):1142-1154.

3. Asselin BL, Gaynon P, Whitlock JA. Recent advances in acute lymphoblastic leukemia in children and adolescents: an expert panel discussion. Curr Opin Oncol. 2013;25(suppl 3):S1-S13.

4. Locatelli F, Schrappe M, Bernardo ME, Rutella S. How I treat relapsed childhood acute lymphoblastic leukemia. Blood. 2012;120(14):2807-2816.

5. Bhojwani D, Pui CH. Relapsed childhood acute lymphoblastic leukaemia. Lancet Oncol. 2013;14(6):e205-e217.

6. Gaynon PS. Childhood acute lymphoblastic leukaemia and relapse. Br J Haematol. 2005;
131(5):579-587.

7. Einsiedel HG, et al. Long-term outcome in children with relapsed ALL by risk-stratified salvage therapy: results of trial acute lymphoblastic leukemia relapse study of the Berlin-Frankfurt-Munster Group 87. J Clin Oncol. 2005;23(31):7942-7950.

8. Uckun FM, Morar S, Qazi S. Vinorelbine-based salvage chemotherapy for therapy-refractory aggressive leukaemias. Brit J Haematol. 2006;135(4):500-508.

9. Tamada K, Chen L. Renewed interest in cancer immunotherapy with the tumor necrosis factor superfamily molecules. Cancer Immunol Immunother. 2006;55(4):355-362.

10. Dimberg LY, Anderson CK, Camidge R, Behbakht K, ThorbumA, Ford HL. On the TRAIL to successful cancer therapy? Predicting and counteracting resistance against TRAIL-based therapeutics. Oncogene. 2013;32(11):1341-1350.

11. Kruyt FA. TRAIL and cancer therapy. Cancer Lett.
2008;263(1):14-25.

12. Bremer E. Targeting of the tumor necrosis receptor superfamily for cancer immunotherapy. ISRN Oncol. 2013;2013:371854.

13. Merino D, Lalaoui N, Morizot A, Solary E, Micheau O. TRAIL in cancer therapy: present and future challenges. Expert Opin Ther Targets. 2007;11(10):1299-1314.

14. Castro Alves C, et al. Leukemia-initiating cells of patient-derived actute lymphoblastic leukemia xenografts are sensitive toward TRAIL. Blood. 2012;119(18):4224-4227.

15. Kelley SK, et al. Preclinical studies to predict the disposition of Apo2L/tumor necrosis factor-related apoptosis-inducing ligand in humans: characterization of in vivo efficacy, pharmacokinetics, and safety. JPharmacol Exp. 2001;299(1):31-38.

16. Duiker EW, et al. The clinical trail of TRAIL. Eur J Cancer. 2006;42(14):2233-2240.

17. Fox NL, Humphreys R, Luster TA, Klein J, Gal- 
lant G. Tumor necrosis factor-related apoptosisinducing ligand (TRAIL) receptor- 1 and receptor-2 agonists for cancer therapy. Expert Opin Biol Ther. 2010;10(1):1-18.

18. Herbst RS, et al. Phase I dose escalation study of recombinant Apo2L/TRAIL, a dual proapoptotic receptor agonist, in patients with cancer. JClin Oncol. 2010;28(17):2839-2846.

19. Soria JC, et al. Randomized phase II study of dulanermin in combination with paclitaxel, carboplatin, and bevacizumab in advanced non-small cell lung cancer. J Clin Oncol. 2011;29(33):4442-4451.

20. de Bruyn M, Bremer E, Helfrich W. Antibodybased fusion proteins to target death receptors in cancer. Cancer Lett. 2010;332(2):175-183.

21. Uckun FM, et al. Autologous bone marrow transplantation in high-risk remission T-lineage acute lymphoblastic leukemia using immunotoxins plus 4-hydroperoxycyclophosphamide for marrow purging. Blood. 1990;76(9):1723-1733.

22. Uckun FM, et al. Detailed studies on expression and function of CD19 surface determinant by using B43 monoclonal antibody and the clinical potential of anti-CD19 immunotoxins. Blood. 1988;71(1):13-29.

23. Uckun FM, et al. Signal transduction through the $\mathrm{CD} 19$ receptor during discrete developmental stages of human B-cell ontogeny. J Biol Chem. 1993;268(28):21172-21184.

24. Kong Y, et al. $\mathrm{CD} 34^{+} \mathrm{CD} 38^{+} \mathrm{CD} 19^{+}$as well as $\mathrm{CD} 34^{+} \mathrm{CD} 38^{-} \mathrm{CD} 19^{+}$cells are leukemia-initiating cells with self-renewal capacity in human B-precursor ALL. Leukemia. 2008;22(6):1207-1213.

25. Le Viseur C, et al. In childhood acute lymphoblastic leukemia, blasts at different stages of immunophenotypic maturation have stem cell properties. Cancer Cell. 2008;14(1):47-58.

26. Varmoor HJ. Malignant stem cells in child- hood acute lymphoblastic leukemia. Cell Cycle. 2009;8(7):996-999.

27. Quintás-Cardama A, Wierda W, O’Brien S. Investigational immunotherapeutics for B-cell malignancies. J Clin Oncol. 2010;28(5):884-892.

28. D'Cruz OJ, Uckun FM. Novel mAb-based therapies for leukemia. In: Uckun FM, ed. Monoclonal Antibodies in Oncology. Uckun London, United Kingdom: Future Medicine Ltd; 2013:54-77.

29. Davilla ML, et al. Efficacy and toxicity management of 19-28z CAR T cell therapy in B cell acute lymphoblastic leukemia. Sci Transl Med. 2014;6(224):224ra25.

30. Von Stackelberg A, et al. A phase $1 / 2$ study of blinatumomab in pediatric patients with relapsed/refractory B-cell precursor acute lymphoblastic leukemia. Blood. 2013;122(21):70.

31. Uckun FM, Sun L, Qazi S, Ma H, Ozer Z. Recombinant human CD19-ligand protein as a potent antileukemic agent. Br J Haematol. 2011; 153(1):15-23.

32. Chen JJ, Knudsen S, Mazin W, Dahlgaard J, Zhang B. A 71-gene signature of TRAIL sensitivity in cancer cells. Mol Cancer Ther. 2012;11(1):34-44.

33. Kim JY, Lee JY, Kim DG, Koo GB, Yu JW, Kim YS. TRADD is critical for resistance to TRAILinduced cell death through NF- $\kappa$ B activation. FEBS Lett. 2011;585(14):2144-2150.

34. MacFarlane M, et al. Mechanisms of resistance to TRAIL-induced apoptosis in primary B cell chronic lymphocytic leukaemia. Oncogene. 2002;21(44):6809-6818.

35. Lemke J, von Karstedt S, Zinngrebe J, Walczak H. Getting TRAIL back on track for cancer therapy. Cell Death Differ. 2014;21(9):1350-1364.

36. Safa AR. c-FLIP, a master anti-apoptotic regulator. Exp Oncol. 2012;34(3):176-184.

37. Clodi K, et al. Expression of tumour necrosis factor (TNF)-related apoptosis-inducing ligand (TRAIL) receptors and sensitivity to TRAIL- induced apoptosis in primary B-cell acute lymphoblastic leukaemia cells. Br J Haematol. 2000;111(2):580-586.

38. Smith M, et al. Uniform approach to risk classification and treatment assignment for children with acute lymphoblastic leukemia. J Clin Oncol. 1996;14(1):18-24.

39. Crowder RN, El-Deiry WS. Caspase-8 regulation of TRAIL-mediated cell death. Exp Oncol. 2012;34(3):160-164.

40. Uckun FM, Qazi S, Ma H, Tuel-Ahlgren L, Ozer Z. STAT3 is a substrate of SYK tyrosine kinase in B-lineage leukemia/lymphoma cells exposed to oxidative stress. Proc Natl Acad Sci U S A. 2010;107(7):2902-2907.

41. Uckun FM, et al. Biphenotypic leukemic lymphocyte precursors in $\mathrm{CD}_{2}{ }^{+} \mathrm{CD} 19^{+}$acute lymphoblastic leukemia and their putative normal counterparts in human fetal hematopoietic tissues. Blood.1989;73(4):1000-1015.

42. Stieglmaier J, et al. Selective induction of apoptosis in leukemic B-lymphoid cells by a CD19specific TRAIL fusion protein. Cancer Immunol Immunother. 2008;57(2):233-246.

43. Wang H, Davis JS, Wu X. Immunoglobulin Fc domain fusion to TRAIL significantly prolongs its plasma half-life and enhances its antitumor activity. Mol Cancer Ther. 2014;13(3):643-650.

44. Uckun FM, Goodman P, Ma H, Dibirdik I, Qazi S. CD22 exon 12 deletion as a novel pathogenic mechanism of human B-precursor leukemia. Proc Natl Acad Sci U S A. 2010;107(39):16852-16857.

45. Uckun FM, et al. Serine phosphorylation by SYK is critical for nuclear localization and transcription factor function of Ikaros. Proc Natl Acad Sci US A. 2012;109(44):18072-18077.

46. Uckun FM, et al. Nanoscale liposomal formulation of a SYK P-site inhibitor against B-precursor leukemia. Blood. 2013;121(21):4348-4354. 